\title{
Asymmetric Iodolactamization Induced by Chiral Oxazolidine Auxiliary
}

\author{
Meihua Shen and Chaozhong Li*
}

Shanghai Institute of Organic Chemistry, Chinese Academy of Sciences, 354 Fenglin Road,

Shanghai 200032, P. R. China

clig@mail.sioc.ac.cn
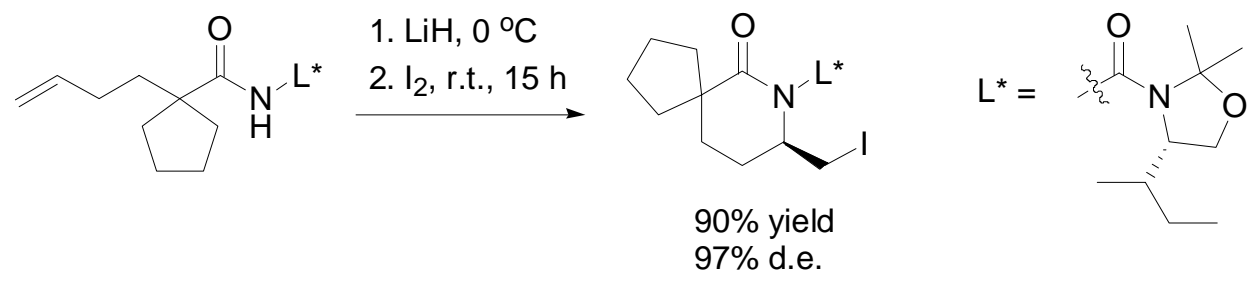

Supporting Information

List of Contents:

1. Characterizations of 1a-d, 4-5, 6a-d and 7-19.

2. ${ }^{1} \mathrm{H}$ NMR spectra of $\mathbf{1 b}, \mathbf{4 - 5}, \mathbf{6 a}, \mathbf{b}, \mathbf{d}, \mathbf{e}, \mathbf{9 - 1 1}, \mathbf{1 3}, \mathbf{1 5 - 1 6}$ and 18.

3. X-ray crystal structure of $\mathbf{6 c}$. 
NMR spectra were recorded in $\mathrm{CDCl}_{3}\left({ }^{1} \mathrm{H}\right.$ at $300 \mathrm{MHz}$ and ${ }^{13} \mathrm{C}$ at $75.47 \mathrm{MHz}$ ) using TMS as the internal standard. All products were isolated by column chromatography on silica gel with hexane - ethyl acetate in an appropriate ratio as the eluent. THF was refluxed with $\mathrm{Na}$ and distilled prior to use. The conditions for ordinary HPLC analysis: Diamonsil-C18 column, size: $4.6 \times 150 \mathrm{~mm}$, UV detector $(220 \mathrm{~nm})$, mobile phase: acetonitrile/water, $60 / 40$ to 100/0. The conditions for chiral HPLC experiments: AD column, UV detector $(205 \mathrm{~nm})$, mobile phase: hexane/isopropanol $=80 / 20$, speed: $0.7 \mathrm{~mL} / \mathrm{min}$.

1a: Colorless oil. $[\alpha]_{\mathrm{D}}{ }^{25}=+4.5\left(\mathrm{c} 1.14, \mathrm{CH}_{3} \mathrm{COCH}_{3}\right) ;{ }^{1} \mathrm{H}$ NMR $\left(300 \mathrm{MHz}, \mathrm{CDCl}_{3}\right) \delta 0.86$ $(3 \mathrm{H}, \mathrm{s}), 0.88(3 \mathrm{H}, \mathrm{s}), 1.18(6 \mathrm{H}, \mathrm{s}), 1.52(3 \mathrm{H}, \mathrm{s}), 1.62(3 \mathrm{H}, \mathrm{s}), 1.89-2.00(1 \mathrm{H}, \mathrm{m}), 2.22-2.36$ $(2 \mathrm{H}, \mathrm{m}), 3.63-3.68(1 \mathrm{H}, \mathrm{m}), 3.81-3.84(1 \mathrm{H}, \mathrm{m}), 3.92-3.97(1 \mathrm{H}, \mathrm{m}), 5.04-5.10(2 \mathrm{H}, \mathrm{m})$, 5.65-5.79 (1H, m), $8.33(1 \mathrm{H}, \mathrm{br}) ;{ }^{13} \mathrm{C} \mathrm{NMR}\left(\mathrm{CDCl}_{3}\right) \delta 18.4,19.3,23.0,24.6,24.7,26.3,30.7$, 42.9, 44.3, 63.2, 65.3, 95.3, 118.6, 133.6, 151.3, 176.1; EIMS: $\mathrm{m} / \mathrm{z}$ (rel intensity) 281 $\left(\mathrm{M}^{+}-\mathrm{CH}_{3}, 8\right), 238$ (30), 210 (5), 155 (8), 128 (100), 100 (49), 83 (88), 70 (18); Anal. calcd for $\mathrm{C}_{16} \mathrm{H}_{28} \mathrm{~N}_{2} \mathrm{O}_{3}: \mathrm{C}, 64.83 ; \mathrm{H}, 9.52 ; \mathrm{N}, 9.45$. Found: $\mathrm{C}, 64.66 ; \mathrm{H}, 9.52 ; \mathrm{N}, 9.45$.

1b: Colorless oil. $[\alpha]_{\mathrm{D}}{ }^{25}=-10.4\left(\mathrm{c} 1.05, \mathrm{CH}_{3} \mathrm{COCH}_{3}\right) ;{ }^{1} \mathrm{H}$ NMR $\left(300 \mathrm{MHz}, \mathrm{CDCl}_{3}\right) \delta 0.91$ $(9 \mathrm{H}, \mathrm{s}), 1.19(6 \mathrm{H}, \mathrm{s}), 1.51(3 \mathrm{H}, \mathrm{s}), 1.68(3 \mathrm{H}, \mathrm{s}), 2.22-2.38(2 \mathrm{H}, \mathrm{m}), 3.68(1 \mathrm{H}, \mathrm{br}), 3.91-4.01$ $(2 \mathrm{H}, \mathrm{m}), 5.06-5.11(2 \mathrm{H}, \mathrm{m}), 5.66-5.80(1 \mathrm{H}, \mathrm{m}), 8.33(1 \mathrm{H}, \mathrm{br}) ;{ }^{13} \mathrm{C} \mathrm{NMR}\left(\mathrm{CDCl}_{3}\right) \delta 23.0$, 24.4, 24.8, 26.6, 27.4, 35.3, 43.0, 44.3, 64.9, 65.8, 96.2, 118.7, 133.6, 153.4, 176.2; EIMS: $\mathrm{m} / z$ (rel intensity) $295\left(\mathrm{M}^{+}-\mathrm{CH}_{3}, 3\right), 253$ (54), 210 (13), 171 (12), 142 (36), 112 (47), 100 (78), 83 (100); HRMS calcd for $\mathrm{C}_{16} \mathrm{H}_{27} \mathrm{~N}_{2} \mathrm{O}_{3}\left(\mathrm{M}^{+}-\mathrm{CH}_{3}\right)$ : 295.2085. Found: 295.2054.

1c: Colorless oil. $[\alpha]_{\mathrm{D}}{ }^{25}=-36.7\left(\mathrm{c} 1.03, \mathrm{CH}_{3} \mathrm{COCH}_{3}\right) ;{ }^{1} \mathrm{H} \mathrm{NMR}\left(300 \mathrm{MHz}, \mathrm{CDCl}_{3}\right) \delta 1.15$ $(3 \mathrm{H}, \mathrm{s}), 1.16(3 \mathrm{H}, \mathrm{s}), 1.55(3 \mathrm{H}, \mathrm{s}), 1.75(3 \mathrm{H}, \mathrm{s}), 2.20-2.34(2 \mathrm{H}, \mathrm{m}), 2.75-2.82(1 \mathrm{H}, \mathrm{m})$, 2.97-3.03 $(1 \mathrm{H}, \mathrm{m}), 3.78-3.81(1 \mathrm{H}, \mathrm{m}), 3.91-3.96(1 \mathrm{H}, \mathrm{m}), 4.15-4.21(1 \mathrm{H}, \mathrm{m}), 5.05-5.11(2 \mathrm{H}$, $\mathrm{m}), 5.66-5.80(1 \mathrm{H}, \mathrm{m}), 7.11-7.31(5 \mathrm{H}, \mathrm{m}), 8.04(1 \mathrm{H}, \mathrm{br}) ;{ }^{13} \mathrm{C} \mathrm{NMR}\left(\mathrm{CDCl}_{3}\right) \delta 23.1,24.5$, 24.7, 26.9, 29.9, 43.0, 44.2, 59.1, 66.8, 95.6, 118.6, 126.7, 128.6, 129.2, 133.6, 137.4, 150.5, 176.4; EIMS: $m / z$ (rel intensity) $329\left(\mathrm{M}^{+}-\mathrm{CH}_{3}, 2\right), 286$ (22), 253 (100), 210 (10), 176 (36), 117 (26), 100 (56), 83 (38); Anal. calcd for $\mathrm{C}_{20} \mathrm{H}_{28} \mathrm{~N}_{2} \mathrm{O}_{3}$ : C, 69.74; $\mathrm{H}, 8.19 ; \mathrm{N}, 8.13$. Found: C, 69.84; H, 8.15; N, 8.10.

1d: Colorless oil. $[\alpha]_{\mathrm{D}}{ }^{25}=+25.2\left(\mathrm{c} 0.78, \mathrm{CH}_{3} \mathrm{COCH}_{3}\right) ;{ }^{1} \mathrm{H}$ NMR $\left(300 \mathrm{MHz}, \mathrm{CDCl}_{3}\right) \delta 0.82$ $(3 \mathrm{H}, \mathrm{d}, J=6.6 \mathrm{~Hz}), 0.86(3 \mathrm{H}, \mathrm{d}, J=6.3 \mathrm{~Hz}), 1.14(3 \mathrm{H}, \mathrm{s}), 1.16(3 \mathrm{H}, \mathrm{s}), 1.26-1.34(1 \mathrm{H}, \mathrm{m})$, 1.40-1.58 (2H, m), $1.49(3 \mathrm{H}, \mathrm{s}), 1.61(3 \mathrm{H}, \mathrm{s}), 2.26(2 \mathrm{H}, \mathrm{d}, J=7.4 \mathrm{~Hz}), 3.73-3.75(1 \mathrm{H}, \mathrm{m})$, $3.97(2 \mathrm{H}, \mathrm{d}, J=8.4 \mathrm{~Hz}), 5.02-5.04(2 \mathrm{H}, \mathrm{m}), 5.64-5.78(1 \mathrm{H}, \mathrm{m}), 8.18(1 \mathrm{H}, \mathrm{br}) ;{ }^{13} \mathrm{C} \mathrm{NMR}$ $\left(\mathrm{CDCl}_{3}\right) \delta 21.6,23.1,23.6,24.5,24.6,25.6,26.7,42.6,43.0,44.2,56.4,67.1,95.0,118.6$, 133.5, 150.1, 176.3; EIMS: $m / z$ (rel intensity) $295\left(\mathrm{M}^{+}-\mathrm{CH}_{3}, 4\right), 252$ (7), 237 (6), 210 (21), 169 (10), 142 (100), 100 (24), 83 (65); Anal. calcd for $\mathrm{C}_{17} \mathrm{H}_{30} \mathrm{~N}_{2} \mathrm{O}_{3}$ : C, 65.77; H, 9.74; N, 9.02. Found: C, 65.64; H, 9.70; N, 8.97.

1-(2,2-Dimethylpent-4-enoyl)-3,3-dimethylurea (4). To a suspension of hexane-washed $\mathrm{NaH}(60 \% \square 3.28 \mathrm{~g} \square 82 \mathrm{mmol})$ in dry THF $(150 \mathrm{~mL})$ was added $N, N$-dimethylurea $(3.52 \mathrm{~g} \square$ $40 \mathrm{mmol}$ ) at room temperature under nitrogen atmosphere. The mixture was stirred for 15 
min at $\mathrm{rt}$ and brought to reflux for $2 \mathrm{~h}$. The solution was then cooled down to $0{ }^{\circ} \mathrm{C}$ and 2,2-dimethyl-4-pentenoic acid chloride was added dropwise. The resulting mixture was stirred at $\mathrm{rt}$ for $2 \mathrm{~h}$ and then refluxed for $15 \mathrm{~h}$. After removal of the solvent, the residue was suspended in ethyl acetate / water and acidified with dilute $\mathrm{HCl}$. The two layers were separated and the aqueous phase was extracted with EtOAc $(3 \times 100 \mathrm{~mL})$. The combined organic layer was washed with brine $(2 \times 100 \mathrm{~mL})$ and then dried over anhydrous $\mathrm{MgSO}_{4}$. After removal of the solvent, the crude product was purified by column chromatography on silica gel using hexane-acetone $(2: 1)$ as the eluent to give pure $\mathbf{4}$ as a yellowish oil. Yield: $6.18 \mathrm{~g}(78 \%)$. IR (film): $v\left(\mathrm{~cm}^{-1}\right) 3242,3079,1663 ;{ }^{1} \mathrm{H} \mathrm{NMR}\left(300 \mathrm{MHz}, \mathrm{CDCl}_{3}\right) \delta 1.22(6 \mathrm{H}$, s), $2.32(2 \mathrm{H}, \mathrm{d} \square J=7.5 \mathrm{~Hz}), 2.96(6 \mathrm{H}, \mathrm{s}), 5.07(1 \mathrm{H}, \mathrm{s}), 5.10-5.13(1 \mathrm{H}, \mathrm{m}), 5.69-5.83(1 \mathrm{H}, \mathrm{m})$, $8.23(1 \mathrm{H}, \mathrm{br}) ;{ }^{13} \mathrm{C} \mathrm{NMR}\left(\mathrm{CDCl}_{3}\right) \delta 24.8,43.1,44.5,118.5,133.7,155.1,176.0$; EIMS: $\mathrm{m} / \mathrm{z}$ (rel intensity) 198 (M+, 34), 183 (6), 157 (4), 115 (34), 83 (44), 72 (100), 55 (58), 44 (52); HRMS calcd for $\mathrm{C}_{10} \mathrm{H}_{18} \mathrm{~N}_{2} \mathrm{O}_{2}: 198.1378$. Found: 198.1373.

Synthesis of 5. General procedure for the iodolactamization reactions with BuLi. To a solution of 4 (99 mg, $0.5 \mathrm{mmol})$ in dry THF (12 mL) was added BuLi (2 M in cyclohexane, $0.25 \mathrm{~mL}, 0.5 \mathrm{mmol}$ ) at $0{ }^{\circ} \mathrm{C}$ under nitrogen atmosphere. The mixture was stirred at $0{ }^{\circ} \mathrm{C}$ for $30 \mathrm{~min}$. Iodine (381 mg, $1.5 \mathrm{mmol}$ ) was added and the resulting mixture was stirred at $\mathrm{rt}$ for $15 \mathrm{~h}$. Aqueous $\mathrm{NaHSO}_{3}(12 \mathrm{~mL})$ was then added slowly. The two layers were separated and the aqueous phase was extracted with ethyl acetate $(3 \times 10 \mathrm{~mL})$. The combined organic layer was washed with brine $(2 \times 10 \mathrm{~mL})$ and then dried over anhydrous $\mathrm{MgSO}_{4}$. After removal of the solvent, the crude product was purified by column chromatography on silica gel using hexane-acetone (4:1) as the eluent to give 5 as a white solid. Yield: $123 \mathrm{mg}$ (76\%). ${ }^{1} \mathrm{H}$ NMR $\left(300 \mathrm{MHz}, \mathrm{CDCl}_{3}\right) \delta 1.19(3 \mathrm{H}, \mathrm{s}), 1.25(3 \mathrm{H}, \mathrm{s}), 1.68-1.78(1 \mathrm{H}, \mathrm{m}), 1.96-2.03(1 \mathrm{H}, \mathrm{m}), 3.03$ $(3 \mathrm{H}, \mathrm{s}), 3.05(3 \mathrm{H}, \mathrm{s}), 3.34-3.38(1 \mathrm{H}, \mathrm{m}), 3.57-3.63(1 \mathrm{H}, \mathrm{m}), 3.83-3.91(1 \mathrm{H}, \mathrm{m}) ;{ }^{13} \mathrm{C} \mathrm{NMR}$ $\left(\mathrm{CDCl}_{3}\right) \delta 12.2,25.0,36.5,39.9,41.2,53.1,153.6,178.8$; EIMS: $m / z$ (rel intensity) $324\left(\mathrm{M}^{+}\right.$, 1), 254 (3), 209 (1), 197 (55), 170 (2), 115 (6), 72 (100), 44 (34); HRMS calcd for $\mathrm{C}_{10} \mathrm{H}_{17} \mathrm{~N}_{2} \mathrm{O}_{2}\left(\mathrm{M}^{+}-\mathrm{I}\right):$ 197.1299. Found: 197.1295.

6a: White solid. The major isomer: ${ }^{1} \mathrm{H} \mathrm{NMR}\left(300 \mathrm{MHz}, \mathrm{CDCl}_{3}\right) \delta 0.86(3 \mathrm{H}, \mathrm{d}, J=6.9 \mathrm{~Hz})$, $0.90(3 \mathrm{H}, \mathrm{d}, J=6.9 \mathrm{~Hz}), 1.17(3 \mathrm{H}, \mathrm{s}), 1.22(3 \mathrm{H}, \mathrm{s}), 1.48-1.56(1 \mathrm{H}, \mathrm{m}), 1.52(3 \mathrm{H}, \mathrm{s}), 1.69(3 \mathrm{H}$, s), 1.96-2.07 $(1 \mathrm{H}, \mathrm{m}), 2.33-2.39(1 \mathrm{H}, \mathrm{m}), 2.88-2.95(1 \mathrm{H}, \mathrm{m}), 3.58(1 \mathrm{H}, \mathrm{dd}, J=3.3,9.0 \mathrm{~Hz})$, 3.86-3.90 $(1 \mathrm{H}, \mathrm{m}), 3.96-4.01(1 \mathrm{H}, \mathrm{m}), 4.05-4.09(1 \mathrm{H}, \mathrm{m}), 4.25-4.35(1 \mathrm{H}, \mathrm{m}) ;{ }^{13} \mathrm{C} \mathrm{NMR}$ $\left(\mathrm{CDCl}_{3}\right) \delta 6.7,17.4,20.0,23.2,24.2,25.7,26.0,30.4,41.5,41.8,55.3,62.4,64.6,96.0$, 150.8, 180.2. The minor isomer: ${ }^{1} \mathrm{H}$ NMR $\left(300 \mathrm{MHz}, \mathrm{CDCl}_{3}\right) \delta 0.82-0.92(6 \mathrm{H}, \mathrm{m}), 1.19(3 \mathrm{H}$, s), $1.20(3 \mathrm{H}, \mathrm{s}), 1.52(3 \mathrm{H}, \mathrm{s}), 1.68(3 \mathrm{H}, \mathrm{s}), 1.83-2.16(3 \mathrm{H}, \mathrm{m}), 3.24-3.31(1 \mathrm{H}, \mathrm{m}), 3.62-3.67$ $(1 \mathrm{H}, \mathrm{m}), 3.83-4.09(3 \mathrm{H}, \mathrm{m}), 4.14-4.18(1 \mathrm{H}, \mathrm{m}) ;{ }^{13} \mathrm{C} \mathrm{NMR}\left(\mathrm{CDCl}_{3}\right) \delta 6.7,18.1,19.8,23.2$, 24.2, 25.7, 26.0, 29.7, 41.5, 41.8, 55.6, 63.1, 64.7, 96.0, 153.8, 177.3. EIMS: $\mathrm{m} / z$ (rel intensity) $407\left(\mathrm{M}^{+}-\mathrm{CH}_{3}, 37\right), 379$ (25), 364 (100), 297 (38), 254 (70), 209 (20), 111 (40), 83 (79); HRMS calcd for $\mathrm{C}_{15} \mathrm{H}_{24} \mathrm{IN}_{2} \mathrm{O}_{3}\left(\mathrm{M}^{+}-\mathrm{CH}_{3}\right)$ : 407.0859. Found: 407.0846.

6b: White solid. The major isomer: ${ }^{1} \mathrm{H}$ NMR $\left(300 \mathrm{MHz}, \mathrm{CDCl}_{3}\right) \delta 0.84(9 \mathrm{H}, \mathrm{s}), 1.10(3 \mathrm{H}, \mathrm{s})$, $1.16(3 \mathrm{H}, \mathrm{s}), 1.36-1.44(1 \mathrm{H}, \mathrm{m}), 1.42(3 \mathrm{H}, \mathrm{s}), 1.69(3 \mathrm{H}, \mathrm{s}), 2.34-2.40(1 \mathrm{H}, \mathrm{m}), 2.80-2.87(1 \mathrm{H}$, $\mathrm{m}), 3.58(1 \mathrm{H}, \mathrm{dd}, J=3.8,13.0 \mathrm{~Hz}), 3.83-4.00(3 \mathrm{H}, \mathrm{m}), 4.25-4.35(1 \mathrm{H}, \mathrm{m}) ;{ }^{13} \mathrm{C} \mathrm{NMR}\left(\mathrm{CDCl}_{3}\right)$ 
$\delta 6.2,23.2,24.0,25.6,26.7,27.9,35.0,41.4,41.6,56.2,65.3,65.9,96.9,153.0,180.7$. The minor isomer: ${ }^{1} \mathrm{H}$ NMR $\left(300 \mathrm{MHz}, \mathrm{CDCl}_{3}\right) \delta 0.84(9 \mathrm{H}, \mathrm{s}), 1.18(3 \mathrm{H}, \mathrm{s}), 1.23(3 \mathrm{H}, \mathrm{s}), 1.47$ $(3 \mathrm{H}, \mathrm{s}), 1.68(3 \mathrm{H}, \mathrm{s}), 2.06-2.12(2 \mathrm{H}, \mathrm{m}), 3.18-3.25(1 \mathrm{H}, \mathrm{m}), 3.48-3.53(1 \mathrm{H}, \mathrm{m}), 3.83-4.00$ $(3 \mathrm{H}, \mathrm{m}), 4.39-4.43(1 \mathrm{H}, \mathrm{m}) ;{ }^{13} \mathrm{C} \mathrm{NMR}\left(\mathrm{CDCl}_{3}\right) \delta 6.2,22.7,24.0,26.1,26.7,27.9,34.8,41.4$, 41.6, 57.2, 64.9, 65.8, 96.9, 156.0, 179.5. EIMS: $m / z$ (rel intensity) $421\left(\mathrm{M}^{+}-\mathrm{CH}_{3}, 10\right), 379$ (100), 297 (83), 280 (23), 254 (23), 209 (16), 170 (11), 83 (34); HRMS calcd for $\mathrm{C}_{16} \mathrm{H}_{26} \mathrm{IN}_{2} \mathrm{O}_{3}\left(\mathrm{M}^{+}-\mathrm{CH}_{3}\right)$ : 421.0912. Found: 421.0950.

6c: White solid. ${ }^{1} \mathrm{H}$ NMR $\left(300 \mathrm{MHz}, \mathrm{CDCl}_{3}\right) \delta 1.14(3 \mathrm{H}, \mathrm{d}, J=1.5 \mathrm{~Hz}), 1.24(3 \mathrm{H}, \mathrm{d}, J=1.5$ $\mathrm{Hz}), 1.47(3 \mathrm{H}, \mathrm{s}), 1.47-1.56(1 \mathrm{H}, \mathrm{m}), 1.72(3 \mathrm{H}, \mathrm{s}), 2.17-2.24(1 \mathrm{H}, \mathrm{m}), 2.69-2.81(2 \mathrm{H}, \mathrm{m})$, $2.99(1 \mathrm{H}, \mathrm{dd}, J=3.9,13.2 \mathrm{~Hz}), 3.34-3.39(1 \mathrm{H}, \mathrm{m}), 3.69-3.72(1 \mathrm{H}, \mathrm{m}), 3.82-3.86(1 \mathrm{H}, \mathrm{m})$, 4.06-4.16 $(1 \mathrm{H}, \mathrm{m}), 4.35-4.41(1 \mathrm{H}, \mathrm{m}), 7.02-7.26(5 \mathrm{H}, \mathrm{m}) ;{ }^{13} \mathrm{C} \mathrm{NMR}\left(\mathrm{CDCl}_{3}\right) \delta 7.8,23.1$, 24.4, 25.6, 27.2, 40.4, 41.4, 41.6, 54.6, 59.2, 66.9, 96.0, 126.8, 128.8, 129.1, 137.5, 149.8, 180.3; EIMS: $m / z$ (rel intensity) $455\left(\mathrm{M}^{+}-\mathrm{CH}_{3}, 6\right), 412$ (11), 379 (100), 297 (64), 280 (36), 254 (21), 159 (20), 83 (52); Anal. calcd for $\mathrm{C}_{20} \mathrm{H}_{27} \mathrm{IN}_{2} \mathrm{O}_{3}$ : C, 51.07; H, 5.79; $\mathrm{N} \square$ 5.96. Found: $\mathrm{C}, 51.39 \mathrm{H}, 5.49 ; \mathrm{N}, 5.70$. The structure was further confirmed by its X-ray diffraction analysis.

6d: White solid. ${ }^{1} \mathrm{H}$ NMR $\left(300 \mathrm{MHz}, \mathrm{CDCl}_{3}\right) \delta 0.79(3 \mathrm{H}, \mathrm{d}, J=6.6 \mathrm{~Hz}), 0.86(3 \mathrm{H}, \mathrm{d}, J=6.6$ $\mathrm{Hz}), 1.12(3 \mathrm{H}, \mathrm{s}), 1.16(3 \mathrm{H}, \mathrm{s}), 1.14-1.23(1 \mathrm{H}, \mathrm{m}), 1.36-1.52(3 \mathrm{H}, \mathrm{m}), 1.46(3 \mathrm{H}, \mathrm{s}), 1.62(3 \mathrm{H}$, s), 2.22-2.29 $(1 \mathrm{H}, \mathrm{m}), 2.90-2.97(1 \mathrm{H}, \mathrm{m}), 3.47(1 \mathrm{H}, \mathrm{dd}, J=3.6,9.3 \mathrm{~Hz}), 3.69-3.72(1 \mathrm{H}, \mathrm{m})$, 3.93-3.97 (1H, m), 4.14-4.20 (2H, m); ${ }^{13} \mathrm{C} \mathrm{NMR}\left(\mathrm{CDCl}_{3}\right) \delta$ 7.4, 21.3, 23.0, 23.7, 24.4, 25.4, 25.7, 26.9, 41.6, 41.8, 43.6, 54.9, 56.2, 67.6, 95.3, 149.5, 180.2; EIMS: $\mathrm{m} / \mathrm{z}$ (rel intensity) $421\left(\mathrm{M}^{+}-\mathrm{CH}_{3}, 32\right), 363$ (4), 336 (100), 280 (34), 252 (29), 209 (46), 156 (20), 98 (45); HRMS calcd for $\mathrm{C}_{16} \mathrm{H}_{26} \mathrm{IN}_{2} \mathrm{O}_{3}\left(\mathrm{M}^{+}-\mathrm{CH}_{3}\right)$ : 421.1056. Found: 421.1022 .

Synthesis of 7. To a solution of $\mathbf{6 a}(84 \mathrm{mg}, 0.2 \mathrm{mmol})$ and $\mathrm{K}_{2} \mathrm{CO}_{3}(83 \mathrm{mg}, 0.6 \mathrm{mmol})$ in DMF $(4 \mathrm{~mL})$ was added $\mathrm{PhSH}(61 \mu \mathrm{L}, 0.6 \mathrm{mmol})$ at $\mathrm{rt}$ under nitrogen atmosphere. The resulting mixture was stirred at $\mathrm{rt}$ for $12 \mathrm{~h}$. Ethyl acetate $(8 \mathrm{~mL})$ and water $(8 \mathrm{~mL})$ were added, and the two layers were separated. The aqueous phase was extracted with ethyl acetate $(2 \times 8 \mathrm{~mL})$. The combined organic layer was washed with brine $(2 \times 10 \mathrm{~mL})$ and dried over anhydrous $\mathrm{MgSO}_{4}$. Excess $\mathrm{PhSH}$ was removed by flash chromatography using hexane as the eluent. The resulting crude product was analyzed by HPLC and further brought to hydrolysis.

A solution of the above crude product in $30 \% \mathrm{KOH}(8 \mathrm{~mL})$ was refluxed for $10 \mathrm{~h}$. Ethyl acetate $(8 \mathrm{~mL})$ was added and the two layers were separated. The aqueous phase was extracted with ethyl acetate $(3 \times 8 \mathrm{~mL})$. The combined organic layer was washed with brine $(2 \times 8 \mathrm{~mL})$ and then dried over anhydrous $\mathrm{MgSO}_{4}$. After removal of the solvent, the crude product was purified by column chromatography on silica gel using hexane-acetone $(2: 1)$ as the eluent to give 7 as a white solid. Yield: $47 \mathrm{mg}(100 \%) .{ }^{1} \mathrm{H} \mathrm{NMR}\left(300 \mathrm{MHz}, \mathrm{CDCl}_{3}\right) \delta$ $1.07(3 \mathrm{H}, \mathrm{s}), 1.13(3 \mathrm{H}, \mathrm{s}), 1.55-1.62(1 \mathrm{H}, \mathrm{m}), 2.03-2.10(1 \mathrm{H}, \mathrm{m}), 2.79-2.86(1 \mathrm{H}, \mathrm{m})$, 2.98-3.04 (1H, m), 3.59-3.68 (1H, m), $6.26(1 \mathrm{H}, \mathrm{br}), 7.15-7.33(5 \mathrm{H}, \mathrm{m}) ;{ }^{13} \mathrm{C} \mathrm{NMR}\left(\mathrm{CDCl}_{3}\right) \delta$ 24.9, 25.3, 40.7 , 40.8, 42.6, 49.9, 126.9, 129.1, 130.5, 134.5, 182.0; EIMS: $m / z$ (rel intensity) $235\left(\mathrm{M}^{+}, 4\right), 149$ (1), 135 (2), 124 (100), 112 (86), 109 (10), 84 (39), 57 (7); Anal. calcd for 
$\mathrm{C}_{13} \mathrm{H}_{17} \mathrm{NOS}$ : C, 66.34; H, 7.28; N, 5.95. Found: C, 66.17; H, 7.18; N, 5.80.

8: Colorless oil. $[\alpha]_{\mathrm{D}}{ }^{25}=-5.6\left(\mathrm{c} 1.00, \mathrm{CH}_{3} \mathrm{COCH}_{3}\right) ;{ }^{1} \mathrm{H} \mathrm{NMR}\left(300 \mathrm{MHz}, \mathrm{CDCl}_{3}\right) \delta$ 0.84-0.89 (6H, m), 0.97-1.10 (1H, m), 1.26-1.37 (1H, m), $1.47(3 \mathrm{H}, \mathrm{s}), 1.61(3 \mathrm{H}, \mathrm{s})$, 1.63-1.71 (1H, m), $2.32(2 \mathrm{H}, \mathrm{q}, J=6.9 \mathrm{~Hz}), 2.74-2.94(2 \mathrm{H}, \mathrm{m}), 3.82-3.92(3 \mathrm{H}, \mathrm{m}), 4.90-5.03$ $(2 \mathrm{H}, \mathrm{m}), 5.71-5.85(1 \mathrm{H}, \mathrm{m}), 8.07(1 \mathrm{H}, \mathrm{br}) ;{ }^{13} \mathrm{C} \mathrm{NMR}\left(\mathrm{CDCl}_{3}\right) \delta 12.1,13.8,23.2,26.17$, 26.24, 28.3, 35.6, 37.0, 61.0, 64.4, 95.3, 115.3, 137.0, 149.7, 175.6; EIMS: $m / z$ (rel intensity) $282\left(\mathrm{M}^{+}-\mathrm{CH}_{3}, 1\right), 267$ (21), 225 (20), 196 (19), 169 (12), 142 (80), 100 (100), 83 (58); Anal. calcd for $\mathrm{C}_{15} \mathrm{H}_{26} \mathrm{~N}_{2} \mathrm{O}_{3}: \mathrm{C}, 63.80 ; \mathrm{H}, 9.28 ; \mathrm{N}, 9.92$. Found: $\mathrm{C}, 63.70 ; \mathrm{H}, 9.38 ; \mathrm{N}, 9.58$.

9: Colorless oil. $[\alpha]_{\mathrm{D}}{ }^{25}=+21.8\left(\mathrm{c} 1.04, \mathrm{CH}_{3} \mathrm{COCH}_{3}\right) ;{ }^{1} \mathrm{H} \mathrm{NMR}\left(300 \mathrm{MHz}, \mathrm{CDCl}_{3}\right) \delta$ 0.86-0.97 (6H, m), 1.08-1.17 (1H, m), $1.16(3 \mathrm{H}, \mathrm{s}), 1.17(3 \mathrm{H}, \mathrm{s}), 1.31-1.44(1 \mathrm{H}, \mathrm{m}), 1.54$ $(3 \mathrm{H}, \mathrm{s}), 1.68(3 \mathrm{H}, \mathrm{s}), 1.75-1.80(1 \mathrm{H}, \mathrm{m}), 2.72-2.86(2 \mathrm{H}, \mathrm{m}), 3.83-3.98(3 \mathrm{H}, \mathrm{m}), 4.94-5.03$ $(2 \mathrm{H}, \mathrm{m}), 5.93-6.02(1 \mathrm{H}, \mathrm{m}), 7.68(1 \mathrm{H}, \mathrm{br}) ;{ }^{13} \mathrm{C} \mathrm{NMR}\left(\mathrm{CDCl}_{3}\right) \delta 12.1,12.9,23.2,26.1,26.4$, 26.9, 27.2, 36.6, 37.1, 47.6, 61.2, 64.3, 95.3, 111.0, 146.9, 149.8, 173.1; EIMS m/z (rel intensity) $310\left(\mathrm{M}^{+}, 3\right), 295$ (21), 253 (20), 224 (23), 171 (8), 142 (91), 100 (100), 83 (52); HRMS calcd for $\mathrm{C}_{17} \mathrm{H}_{31} \mathrm{~N}_{2} \mathrm{O}_{3}\left(\mathrm{M}^{+}+1\right)$ : 311.2329 . Found: 311.2343 .

10: Colorless oil. $[\alpha]_{\mathrm{D}}{ }^{25}=-21.4\left(\mathrm{c} 0.88, \mathrm{CH}_{3} \mathrm{COCH}_{3}\right) ;{ }^{1} \mathrm{H}$ NMR $\left(300 \mathrm{MHz}, \mathrm{CDCl}_{3}\right) \delta$ 0.88-0.97 $(6 \mathrm{H}, \mathrm{m}), 1.05-1.17(1 \mathrm{H}, \mathrm{m}), 1.33-1.44(1 \mathrm{H}, \mathrm{m}), 1.54(3 \mathrm{H}, \mathrm{s}), 1.69(3 \mathrm{H}, \mathrm{s})$, 1.72-1.80 (3H, m), 2.08-2.15 (2H, m), 2.71-2.91 (2H, m), 3.89-3.99 (3H, m), 4.97-5.07 (2H, $\mathrm{m}), 5.74-5.88(1 \mathrm{H}, \mathrm{m}), 8.02(1 \mathrm{H}, \mathrm{br}) ;{ }^{13} \mathrm{C} \mathrm{NMR}\left(\mathrm{CDCl}_{3}\right) \delta 12.1,13.8,23.2,23.5,26.19$, 26.25, 33.0, 35.7, 37.1, 61.0, 64.4, 95.3, 115.0, 137.9, 149.7, 176.2; EIMS: $m / z$ (rel intensity) 296 ( $\left.\mathrm{M}^{+}, 1\right), 281$ (20), 239 (18), 210 (15), 197 (6), 142 (71), 100 (72), 84 (100); HRMS calcd for $\mathrm{C}_{16} \mathrm{H}_{29} \mathrm{~N}_{2} \mathrm{O}_{3}\left(\mathrm{M}^{+}+1\right)$ : 297.2173. Found 297.2189.

11: Colorless oil. $[\alpha]_{\mathrm{D}}{ }^{25}=-3.4\left(\mathrm{c} 1.08, \mathrm{CH}_{3} \mathrm{COCH}_{3}\right) ;{ }^{1} \mathrm{H}$ NMR $\left(300 \mathrm{MHz}, \mathrm{CDCl}_{3}\right) \delta$ 0.82-0.90 (6H, m), 0.96-1.07 (1H, m), $1.17(6 \mathrm{H}, \mathrm{s}), 1.22-1.31(1 \mathrm{H}, \mathrm{m}), 1.51(3 \mathrm{H}, \mathrm{s})$, 1.58-1.71 (3H, m), $1.62(3 \mathrm{H}, \mathrm{s}), 1.95-2.02(2 \mathrm{H}, \mathrm{m}), 3.78-3.82(2 \mathrm{H}, \mathrm{m}), 3.92-3.98(1 \mathrm{H}, \mathrm{m})$, 4.88-4.99 (2H, m), 5.66-5.80 (1H, m), $8.43(1 \mathrm{H}, \mathrm{br}) ;{ }^{13} \mathrm{C} \mathrm{NMR}\left(\mathrm{CDCl}_{3}\right) \delta 12.1,14.5,23.1$, 24.8, 25.0, 25.9, 26.1, 28.9, 37.2, 39.4, 42.9, 62.0, 65.0, 95.4, 114.6, 137.9, 151.4, 176.3; EIMS: $m / z$ (rel intensity) $309\left(\mathrm{M}^{+}-\mathrm{CH}_{3}, 4\right), 267$ ( 7), 238 (14), 212 ( 5), 169 ( 8), 142 (87), 113 (71), 100 (100); HRMS calcd for $\mathrm{C}_{18} \mathrm{H}_{32} \mathrm{~N}_{2} \mathrm{O}_{3} \mathrm{Na}\left(\mathrm{M}^{+}+\mathrm{Na}\right)$ : 347.2305. Found 347.2316.

12: Colorless oil. $[\alpha]_{\mathrm{D}}{ }^{25}=+14.9\left(\mathrm{c} 1.01, \mathrm{CH}_{3} \mathrm{COCH}_{3}\right) ;{ }^{1} \mathrm{H}$ NMR $\left(300 \mathrm{MHz}, \mathrm{CDCl}_{3}\right) \delta$ 0.86-0.94 (6H, m), 0.98-1.14 (1H, m), 1.26-1.81 (12H, m), $1.57(3 \mathrm{H}, \mathrm{s}), 1.69(3 \mathrm{H}, \mathrm{m})$, 1.94-2.07 (4H, m), 3.84-3.90 (2H, m), 3.98-4.04 (1H, m), 4.94-5.05 (2H, m), 5.70-5.83 $(1 \mathrm{H}$, m), $7.61(1 \mathrm{H}, \mathrm{br}) ;{ }^{13} \mathrm{C} \mathrm{NMR}\left(\mathrm{CDCl}_{3}\right) \delta 12.1,14.3,22.8,22.9,23.1,25.7,26.0,26.2,28.0$, 33.6, 34.0, 37.7, 38.9, 47.4, 62.3, 65.0, 95.4, 114.7, 137.9, 150.9, 175.2; EIMS: $m / z$ (rel intensity) $349\left(\mathrm{M}^{+}-\mathrm{CH}_{3}, 3\right), 306$ (8), 278 (15), 252 (8), 208 (4), 182 (9), 153 (99), 100 (100); Anal. calcd for $\mathrm{C}_{21} \mathrm{H}_{36} \mathrm{~N}_{2} \mathrm{O}_{3}$ : C, 69.19; H, 9.95; N, 7.68. Found: C, 69.03; H, 10.06; N, 7.60.

13: Colorless oil. $[\alpha]_{\mathrm{D}}{ }^{25}=+14.2\left(\mathrm{c} 1.14, \mathrm{CH}_{3} \mathrm{COCH}_{3}\right) ;{ }^{1} \mathrm{H}$ NMR $\left(300 \mathrm{MHz}, \mathrm{CDCl}_{3}\right) \delta$ 0.89-0.94 (6H, m), 1.01-1.13 (1H, m), 1.31-1.42 (1H, m), 1.51-1.81 (9H, m), $1.56(3 \mathrm{H}, \mathrm{s})$, 
$1.68(3 \mathrm{H}, \mathrm{s}), 1.96-2.18(4 \mathrm{H}, \mathrm{m}), 3.81-3.89(2 \mathrm{H}, \mathrm{m}), 3.97-4.02(1 \mathrm{H}, \mathrm{m}), 4.94-5.05(2 \mathrm{H}, \mathrm{m})$, 5.71-5.85 (1H, m), $7.46(1 \mathrm{H}, \mathrm{br}) ;{ }^{13} \mathrm{C} \mathrm{NMR}\left(\mathrm{CDCl}_{3}\right) \delta 12.2,14.4,23.2,24.7,24.8,26.1,26.4$, 29.8, 35.5, 35.8, 37.5, 38.7, 55.3, 62.1, 64.9, 95.5, 114.9, 137.7, 150.3, 175.5; EIMS: $\mathrm{m} / z$ (rel intensity) $335\left(\mathrm{M}^{+}-\mathrm{CH}_{3}, 2\right), 264$ (10), 238 (5), 169 (8), 142 (78), 139 (100), 123 (38), 100 (100); HRMS calcd for $\mathrm{C}_{20} \mathrm{H}_{34} \mathrm{~N}_{2} \mathrm{O}_{3} \mathrm{Na}\left(\mathrm{M}^{+}+\mathrm{Na}\right)$ : 373.2462. Found: 373.2465.

14: White solid. The major isomer: ${ }^{1} \mathrm{H}$ NMR $\left(300 \mathrm{MHz}, \mathrm{CDCl}_{3}\right) \delta 0.87-0.92(6 \mathrm{H}, \mathrm{m})$, $1.06-1.33(3 \mathrm{H}, \mathrm{m}), 1.53(3 \mathrm{H}, \mathrm{s}), 1.65-1.85(2 \mathrm{H}, \mathrm{m}), 1.70(3 \mathrm{H}, \mathrm{s}), 2.43-2.59(2 \mathrm{H}, \mathrm{m})$, 2.96-3.02 (1H, m), $3.54(1 \mathrm{H}, \mathrm{dd}, J=3.3,12.3 \mathrm{~Hz}), 3.84-3.89(1 \mathrm{H}, \mathrm{m}), 3.94-4.03(1 \mathrm{H}, \mathrm{m})$, 4.25-4.30 (1H, m), 4.33-4.42 (1H, m); ${ }^{13} \mathrm{C} \mathrm{NMR}\left(\mathrm{CDCl}_{3}\right) \delta 7.3,12.1,14.4,23.4,25.7,26.4$, $26.8,31.7,37.1,58.2,60.5,64.4,96.0,150.6,175.2$. The minor isomer: ${ }^{1} \mathrm{H}$ NMR $(300 \mathrm{MHz}$, $\left.\mathrm{CDCl}_{3}\right) \delta$ 0.87-0.92 (6H, m), 1.06-1.33 (3H, m), $1.55(3 \mathrm{H}, \mathrm{s}), 1.65-1.85(2 \mathrm{H}, \mathrm{m}), 1.68(3 \mathrm{H}$, s), 2.18-2.24 (2H, m), 3.37-3.43 (1H, m), 3.83-4.13 (5H, m); ${ }^{13} \mathrm{C} \mathrm{NMR}\left(\mathrm{CDCl}_{3}\right) \delta 9.3,12.2$, 14.7, 23.3, 25.5, 25.9, 26.8, 29.6, 36.8, 58.2, 60.4, 61.1, 95.8, 151.1, 170.3. EIMS: $\mathrm{m} / z$ (rel intensity) $393\left(\mathrm{M}^{+}-\mathrm{CH}_{3}, 38\right), 351$ (37), 322 (100), 269 (83), 252 (46), 226 (75), 142 (98), 83 (90); Anal. calcd for $\mathrm{C}_{14} \mathrm{H}_{22} \mathrm{IN}_{2} \mathrm{O}_{3}$ : C, 44.13; H, 6.17; N, 6.86. Found: C, 43.94; H, 6.13; N, 6.76.

15: White solid. The major isomer: ${ }^{1} \mathrm{H}$ NMR $\left(300 \mathrm{MHz}, \mathrm{CDCl}_{3}\right) \delta 0.86-0.93(6 \mathrm{H}, \mathrm{m})$, 1.06-1.34 (2H, m), $1.19(3 \mathrm{H}, \mathrm{s}), 1.42(3 \mathrm{H}, \mathrm{s}), 1.52(3 \mathrm{H}, \mathrm{s}), 1.72(3 \mathrm{H}, \mathrm{s}), 1.75-1.82(1 \mathrm{H}, \mathrm{m})$, $2.37(2 \mathrm{H}, \mathrm{d}, J=1.8 \mathrm{~Hz}), 3.04-3.11(1 \mathrm{H}, \mathrm{m}), 3.38(1 \mathrm{H}, \mathrm{dd}, J=3.9,11.7 \mathrm{~Hz}), 3.87(1 \mathrm{H}, \mathrm{dd}, J=$ 2.4, $6.0 \mathrm{~Hz}), 3.98-4.03(1 \mathrm{H}, \mathrm{m}), 4.25-4.30(2 \mathrm{H}, \mathrm{m}) ;{ }^{13} \mathrm{C} \mathrm{NMR}\left(\mathrm{CDCl}_{3}\right) \delta-1.9,12.3,14.2$, $22.2,23.2,25.6,26.8,28.5,36.0,37.5,47.9,60.9,64.2,65.6,96.0,150.8,173.1$. The minor isomer: ${ }^{1} \mathrm{H}$ NMR $\left(300 \mathrm{MHz}, \mathrm{CDCl}_{3}\right) \delta$ 0.86-0.93 (6H, m), 1.06-1.34 (2H, m), $1.20(3 \mathrm{H}, \mathrm{s})$, $1.27(3 \mathrm{H}, \mathrm{s}), 1.58(3 \mathrm{H}, \mathrm{s}), 1.75-1.82(1 \mathrm{H}, \mathrm{m}), 1.80(3 \mathrm{H}, \mathrm{s}), 2.05-2.15(1 \mathrm{H}, \mathrm{m}), 2.43(2 \mathrm{H}, \mathrm{s})$, 2.69-2.75 $(1 \mathrm{H}, \mathrm{m}), 3.58-3.66(2 \mathrm{H}, \mathrm{m}), 3.94-4.03(2 \mathrm{H}, \mathrm{m}) ;{ }^{13} \mathrm{C} \mathrm{NMR}\left(\mathrm{CDCl}_{3}\right) \delta-1.9,12.3$, 14.4, 21.8, 22.8, 25.9, 27.1, 29.4, 36.0, 37.5, 45.3, 61.6, 64.5, 68.2, 96.0, 153.2, 171.8. EIMS: $\mathrm{m} / z$ (rel intensity) $421\left(\mathrm{M}^{+}-\mathrm{CH}_{3}, 39\right), 379$ (38), 350 (100), 297 (88), 280 (47), 254 (64), 142 (62), 83 (76); HRMS calcd for $\mathrm{C}_{17} \mathrm{H}_{29} \mathrm{IN}_{2} \mathrm{O}_{3} \mathrm{Na}\left(\mathrm{M}^{+}+\mathrm{Na}\right)$ : 459.1115. Found: 459.1121.

16: White solid. The major isomer: ${ }^{1} \mathrm{H}$ NMR $\left(300 \mathrm{MHz}, \mathrm{CDCl}_{3}\right) \delta 0.87-0.96(6 \mathrm{H}, \mathrm{m})$, 1.12-1.36 (2H, m), $1.57(3 \mathrm{H}, \mathrm{s}), 1.64-1.99(4 \mathrm{H}, \mathrm{m}), 1.73(3 \mathrm{H}, \mathrm{s}), 2.33-2.53(3 \mathrm{H}, \mathrm{m})$, 2.48-2.53 (2H, m), 2.93-3.00 (1H, m), 3.37-3.40 (1H, m), 3.81-3.97 $(3 \mathrm{H}, \mathrm{m}), 4.15-4.21(1 \mathrm{H}$, $\mathrm{m}) ;{ }^{13} \mathrm{C} \mathrm{NMR}\left(\mathrm{CDCl}_{3}\right) \delta 6.2,12.4,14.2,19.3,21.8,25.4,27.0,28.8,33.4,38.4,57.7,59.8$, 63.2, 96.3, 153.6, 171.8. The minor isomer: ${ }^{1} \mathrm{H}$ NMR (300 MHz, $\left.\mathrm{CDCl}_{3}\right) \delta 0.87-0.96(6 \mathrm{H}$, m), 1.10-1.36 (2H, m), $1.62(3 \mathrm{H}, \mathrm{s}), 1.64-1.99(4 \mathrm{H}, \mathrm{m}), 1.76(3 \mathrm{H}, \mathrm{s}), 2.33-2.53(3 \mathrm{H}, \mathrm{m})$, 2.48-2.53 (2H, m), 3.20-3.27 $(1 \mathrm{H}, \mathrm{m}), 3.55-3.58(1 \mathrm{H}, \mathrm{m}), 3.81-3.97(3 \mathrm{H}, \mathrm{m}), 4.35-4.41(1 \mathrm{H}$, $\mathrm{m}) ;{ }^{13} \mathrm{C} \mathrm{NMR}\left(\mathrm{CDCl}_{3}\right) \delta$ 6.6, 12.4, 16.0, 19.3, 22.0, 25.1, 26.7, 29.7, 32.0, 38.3, 57.7, 59.8, 62.7, 96.0, 155.8, 170.1. EIMS: $\mathrm{m} / z$ (rel intensity) $407\left(\mathrm{M}^{+}-\mathrm{CH}_{3}, 7\right), 365$ (10), 336 (17), 307 (5), 240 (14), 142 (25), 100 (20), 84 (100); HRMS calcd for $\mathrm{C}_{16} \mathrm{H}_{27} \mathrm{IN}_{2} \mathrm{O}_{3} \mathrm{Na}\left(\mathrm{M}^{+}+\mathrm{Na}\right)$ : 445.0959. Found: 445.0963.

17: White solid. The major isomer: ${ }^{1} \mathrm{H}$ NMR $\left(300 \mathrm{MHz}, \mathrm{CDCl}_{3}\right) \delta 0.86-0.96(6 \mathrm{H}, \mathrm{m})$, 1.00-1.22 (2H, m), $1.18(3 \mathrm{H}, \mathrm{s}), 1.20(3 \mathrm{H}, \mathrm{s}), 1.55(3 \mathrm{H}, \mathrm{s}), 1.66-1.82(4 \mathrm{H}, \mathrm{m}), 1.70(3 \mathrm{H}, \mathrm{s})$, 
2.32-2.41 $(1 \mathrm{H}, \mathrm{m}), 2.90-2.96(1 \mathrm{H}, \mathrm{m}), 3.34-3.37(1 \mathrm{H}, \mathrm{m}), 3.69-3.95(3 \mathrm{H}, \mathrm{m}), 4.05-4.20(1 \mathrm{H}$, $\mathrm{m}) ;{ }^{13} \mathrm{C} \mathrm{NMR}\left(\mathrm{CDCl}_{3}\right) \delta$ 6.6, 12.4, 13.1, 21.7, 25.3, 25.6, 26.2, 26.6, 27.0, 34.0, 38.1, 38.8, 58.3, 61.1, 63.3, 96.3, 154.3, 178.3. The minor isomer: ${ }^{1} \mathrm{H}$ NMR $\left(300 \mathrm{MHz}, \mathrm{CDCl}_{3}\right) \delta$ 0.86-0.96 (6H, m), 1.00-1.22 (2H, m), $1.18(3 \mathrm{H}, \mathrm{s}), 1.20(3 \mathrm{H}, \mathrm{s}), 1.55(3 \mathrm{H}, \mathrm{s}), 1.66-1.82(4 \mathrm{H}$, $\mathrm{m}), 1.70(3 \mathrm{H}, \mathrm{s}), 1.95-2.04(1 \mathrm{H}, \mathrm{m}), 3.15-3.21(1 \mathrm{H}, \mathrm{m}), 3.69-3.95(4 \mathrm{H}, \mathrm{m}), 4.40-4.49(1 \mathrm{H}$, $\mathrm{m}) ;{ }^{13} \mathrm{C} \mathrm{NMR}\left(\mathrm{CDCl}_{3}\right) \delta$ 6.8, 12.4, 13.6, 22.2, 25.4, 25.7, 26.3, 26.8, 27.6, 34.0, 38.2, 38.8, 58.4, 62.2, 64.1, 96.0, 154.1, 176.3. EIMS: $m / z$ (rel intensity) $435\left(\mathrm{M}^{+}-\mathrm{CH}_{3}, 20\right), 393$ (32), 364 (52), 294 (53), 266 (100), 224 (14), 140 (20), 95 (51); Anal. calcd for $\mathrm{C}_{18} \mathrm{H}_{31} \mathrm{IN}_{2} \mathrm{O}_{3}$ : C, 48.00; H, 6.94; N, 6.22. Found: C, 47.92; H, 6.94; N, 5.96.

18: White solid. The major isomer: ${ }^{1} \mathrm{H}$ NMR $\left(300 \mathrm{MHz}, \mathrm{CDCl}_{3}\right) \delta 0.83-0.97(6 \mathrm{H}, \mathrm{m})$, 1.04-1.82 (14H, m), $1.59(3 \mathrm{H}, \mathrm{s}), 1.73(3 \mathrm{H}, \mathrm{s}), 2.00-2.07(2 \mathrm{H}, \mathrm{m}), 2.30-2.35(1 \mathrm{H}, \mathrm{m})$, 2.92-2.98 (1H, m), $3.35(1 \mathrm{H}, \mathrm{dd}, J=2.8,9.2 \mathrm{~Hz}), 3.70-3.73(1 \mathrm{H}, \mathrm{m}), 3.83-3.98(2 \mathrm{H}, \mathrm{m})$, 4.13-4.20 (1H, m); ${ }^{13} \mathrm{C} \mathrm{NMR}\left(\mathrm{CDCl}_{3}\right) \delta 6.7,12.4,13.0,20.9,21.1,21.7,24.7,25.3,25.5$, 27.0, 27.8, 33.1, 33.6, 38.1, 42.2, 57.5, 61.2, 63.3, 96.3, 154.5, 178.6. The minor isomer: ${ }^{1} \mathrm{H}$ NMR (300 MHz, $\left.\mathrm{CDCl}_{3}\right) \delta$ 0.83-0.97 $(6 \mathrm{H}, \mathrm{m}), 1.04-1.82(20 \mathrm{H}, \mathrm{m}), 2.00-2.07(2 \mathrm{H}, \mathrm{m})$, 2.30-2.35 $(1 \mathrm{H}, \mathrm{m}), 2.92-2.98(1 \mathrm{H}, \mathrm{m}), 3.15-3.21(1 \mathrm{H}, \mathrm{m}), 3.41-3.46(1 \mathrm{H}, \mathrm{m}), 3.70-3.98(2 \mathrm{H}$, m), 4.42-4.49 $(1 \mathrm{H}, \mathrm{m}) ;{ }^{13} \mathrm{C} \mathrm{NMR}\left(\mathrm{CDCl}_{3}\right) \delta 7.0,12.4,13.7,20.6,20.9,22.0,24.1,25.2,25.4$, 26.6, 27.7, 32.6, 33.5, 38.2, 42.2, 57.5, 61.2, 64.0, 96.0, 154.3, 176.4. EIMS: $\mathrm{m} / \mathrm{z}$ (rel intensity) $475\left(\mathrm{M}^{+}-\mathrm{CH}_{3}, 6\right), 433$ (12), 404 (21), 308 (27), 306 (100), 180 (13), 156 (15), 135 (16); HRMS calcd for $\mathrm{C}_{21} \mathrm{H}_{36} \mathrm{IN}_{2} \mathrm{O}_{3}\left(\mathrm{M}^{+}+1\right)$ : 491.1765. Found: 491.1764 .

19: White solid. The major isomer: ${ }^{1} \mathrm{H}$ NMR $\left(300 \mathrm{MHz}, \mathrm{CDCl}_{3}\right) \delta 0.87-0.97(6 \mathrm{H}, \mathrm{m})$, $1.12-1.37(2 \mathrm{H}, \mathrm{m}), 1.57(3 \mathrm{H}, \mathrm{s}), 1.62-1.82(11 \mathrm{H}, \mathrm{m}), 1.71(3 \mathrm{H}, \mathrm{s}), 2.35-2.41(2 \mathrm{H}, \mathrm{m})$, 2.88-2.95 $(1 \mathrm{H}, \mathrm{m}), 3.36(1 \mathrm{H}, \mathrm{dd}, J=2.7,9.3 \mathrm{~Hz}), 3.69-3.72(1 \mathrm{H}, \mathrm{m}), 3.81-3.85(1 \mathrm{H}, \mathrm{m})$, 3.91-3.96 (1H, m), 4.12-4.18 (1H, m); ${ }^{13} \mathrm{C} \mathrm{NMR}\left(\mathrm{CDCl}_{3}\right) \delta$ 6.7, 12.4, 13.1, 21.8, 25.4, 25.6, 26.7, 27.1, 32.9, 36.6, 38.1, 39.0, 49.9, 58.0, 61.2, 63.4, 96.4, 154.4 178.4. The minor isomer: ${ }^{1} \mathrm{H}$ NMR $\left(300 \mathrm{MHz}, \mathrm{CDCl}_{3}\right) \delta$ 0.87-0.97 (6H, m), 1.12-1.37 (2H, m), $1.57(3 \mathrm{H}, \mathrm{s}), 1.62-1.82$ $(11 \mathrm{H}, \mathrm{m}), 1.72(3 \mathrm{H}, \mathrm{s}), 2.35-2.41(2 \mathrm{H}, \mathrm{m}), 3.28-3.35(1 \mathrm{H}, \mathrm{m}), 3.42-3.46(1 \mathrm{H}, \mathrm{m}), 3.69-3.96$ $(3 \mathrm{H}, \mathrm{m}), 4.38-4.45(1 \mathrm{H}, \mathrm{m}) ;{ }^{13} \mathrm{C} \mathrm{NMR}\left(\mathrm{CDCl}_{3}\right) \delta 7.1,11.8,12.7,22.1,24.5,25.7,26.7,27.1$, 31.7, 36.5, 38.0, 38.4, 49.6, 57.9, 62.1, 64.3, 95.6, 154.3 176.4. EIMS: $m / z$ (rel intensity) $461\left(\mathrm{M}^{+}-\mathrm{CH}_{3}, 9\right), 419$ (18), 390 (33), 320 (22), 292 (100), 184 (10), 156 (76), 142 (12); Anal. calcd for $\mathrm{C}_{20} \mathrm{H}_{33} \mathrm{IN}_{2} \mathrm{O}_{3}:$ C, 50.42; H, 6.98; N, 5.88. Found: C, 50.43; H, 6.81; N, 5.76. 


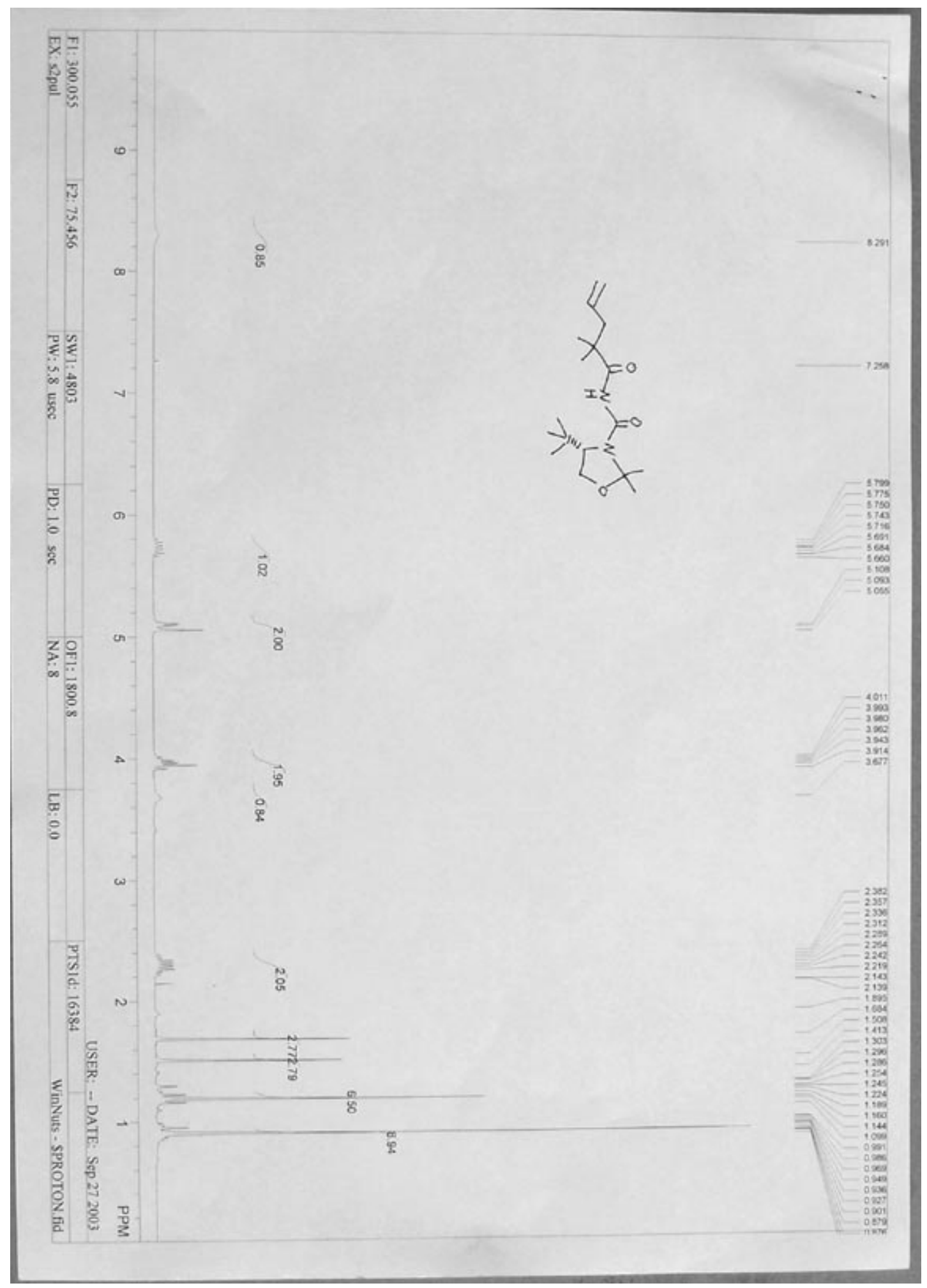




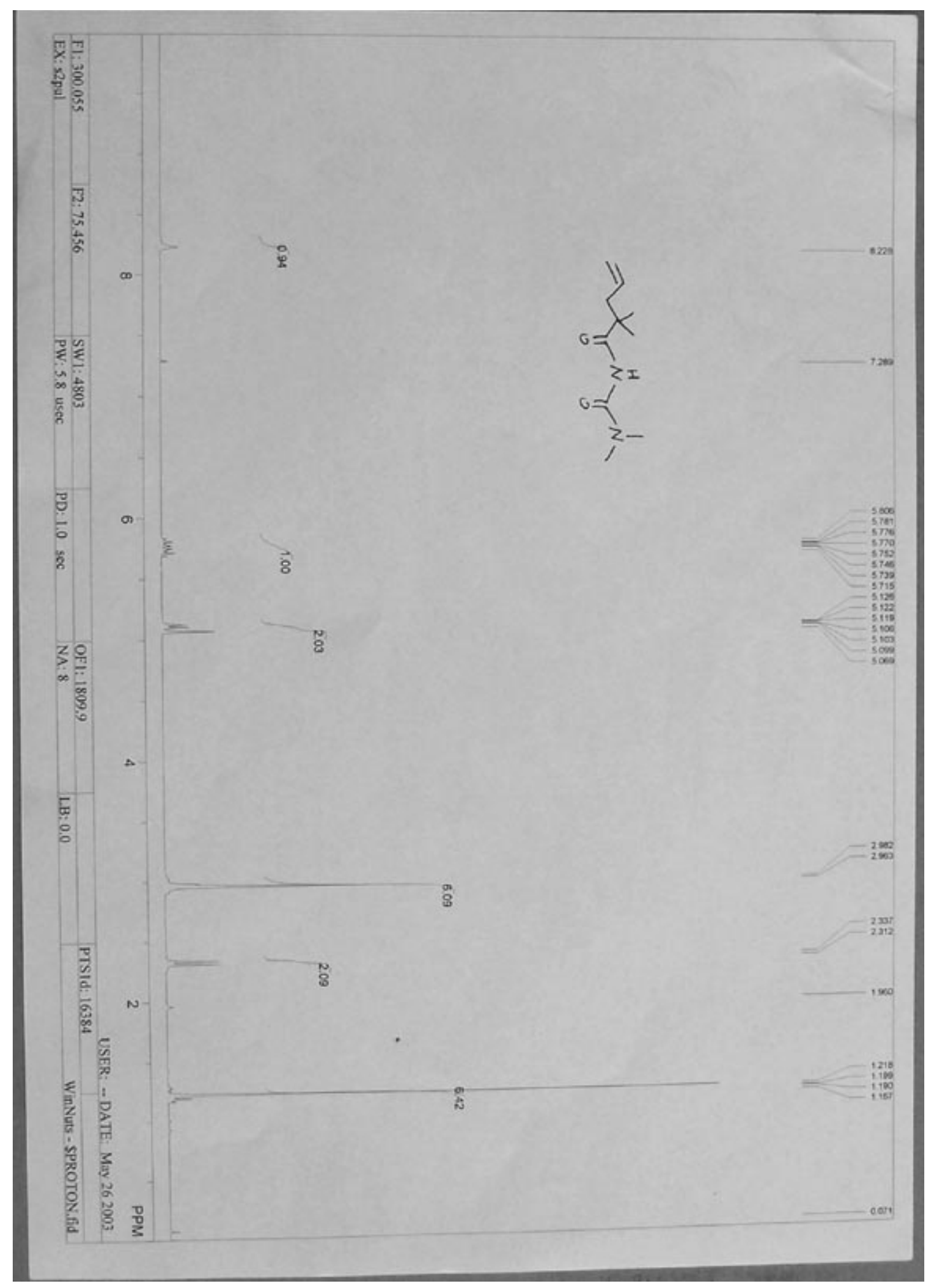




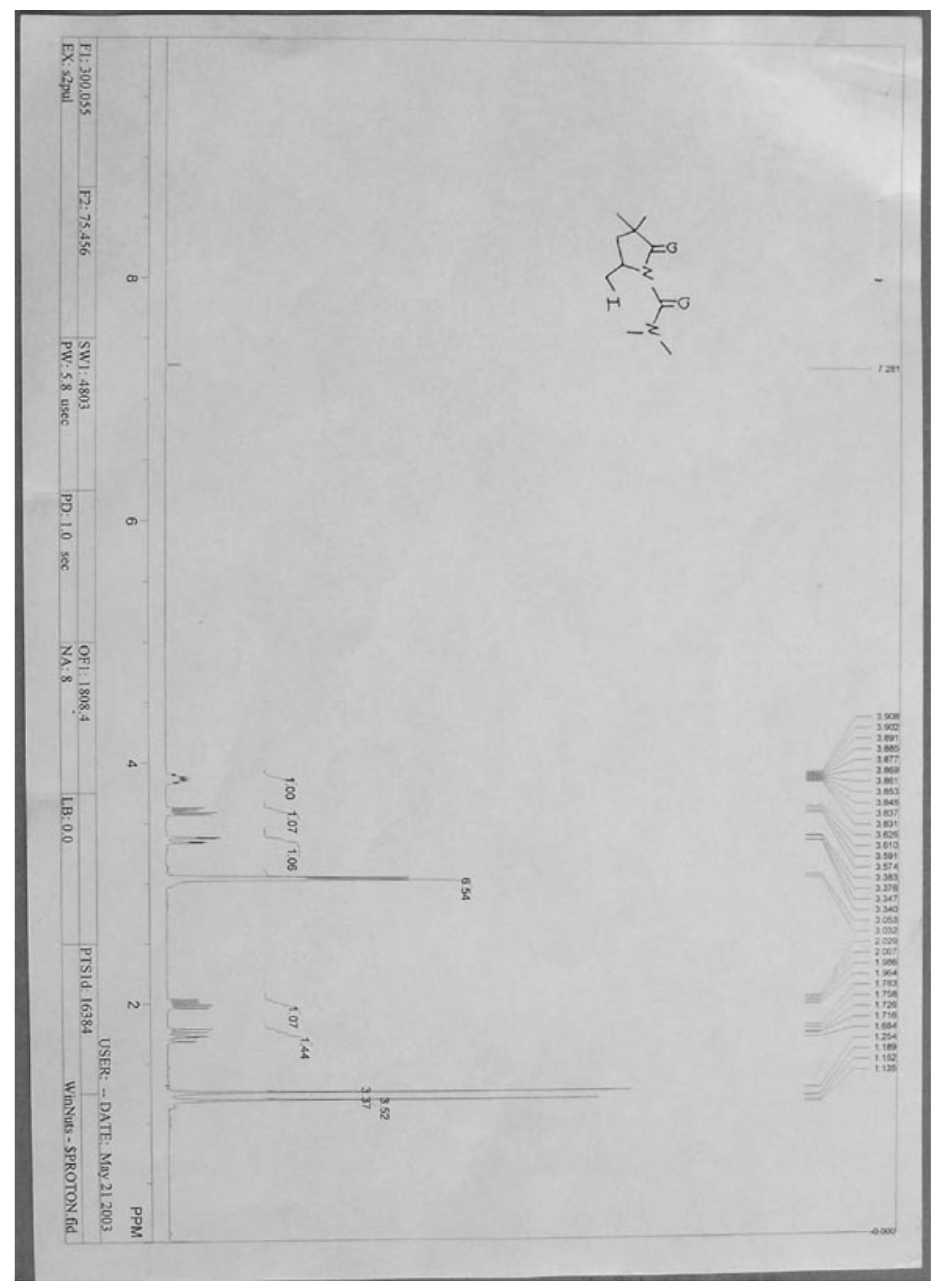




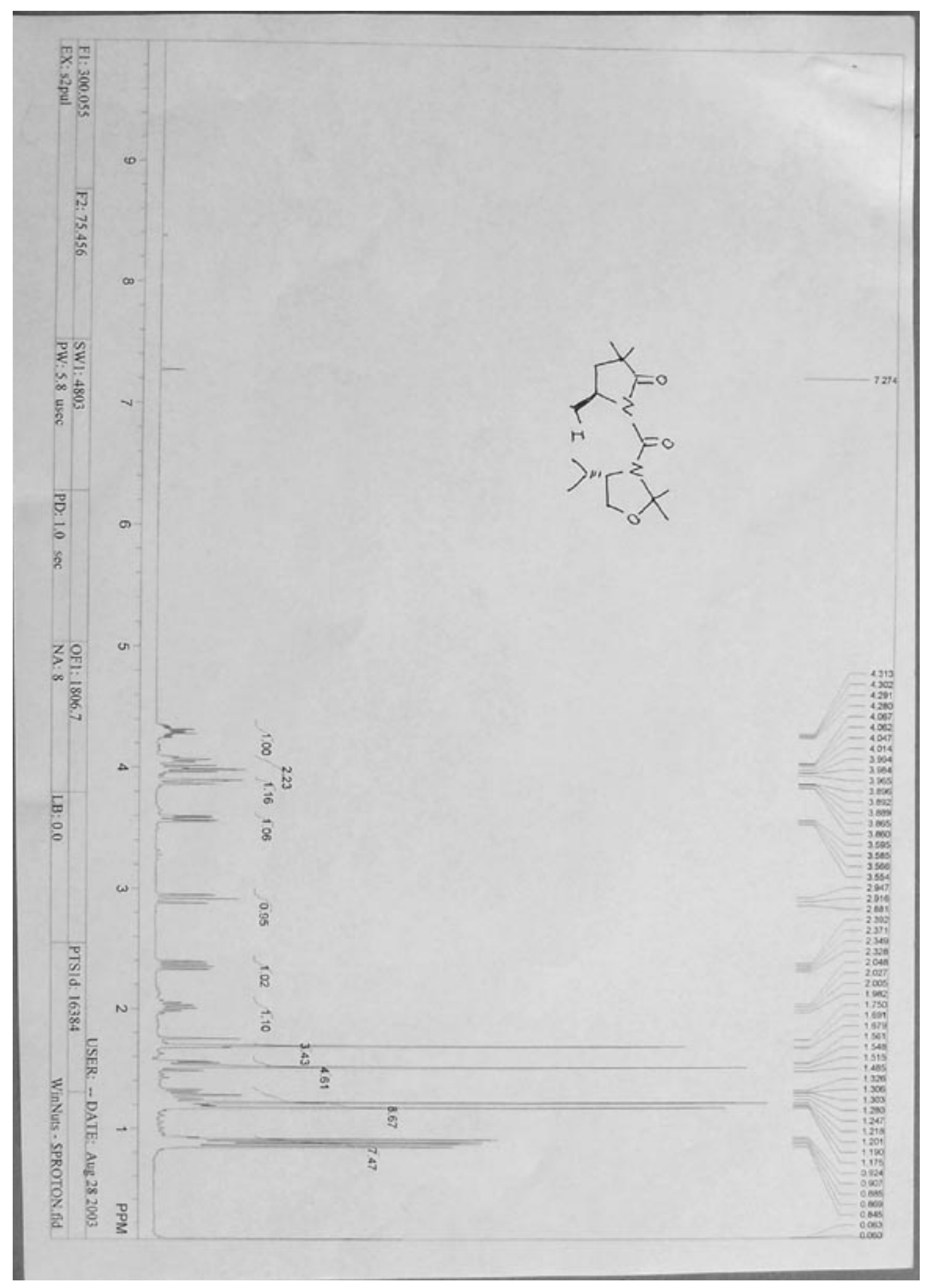




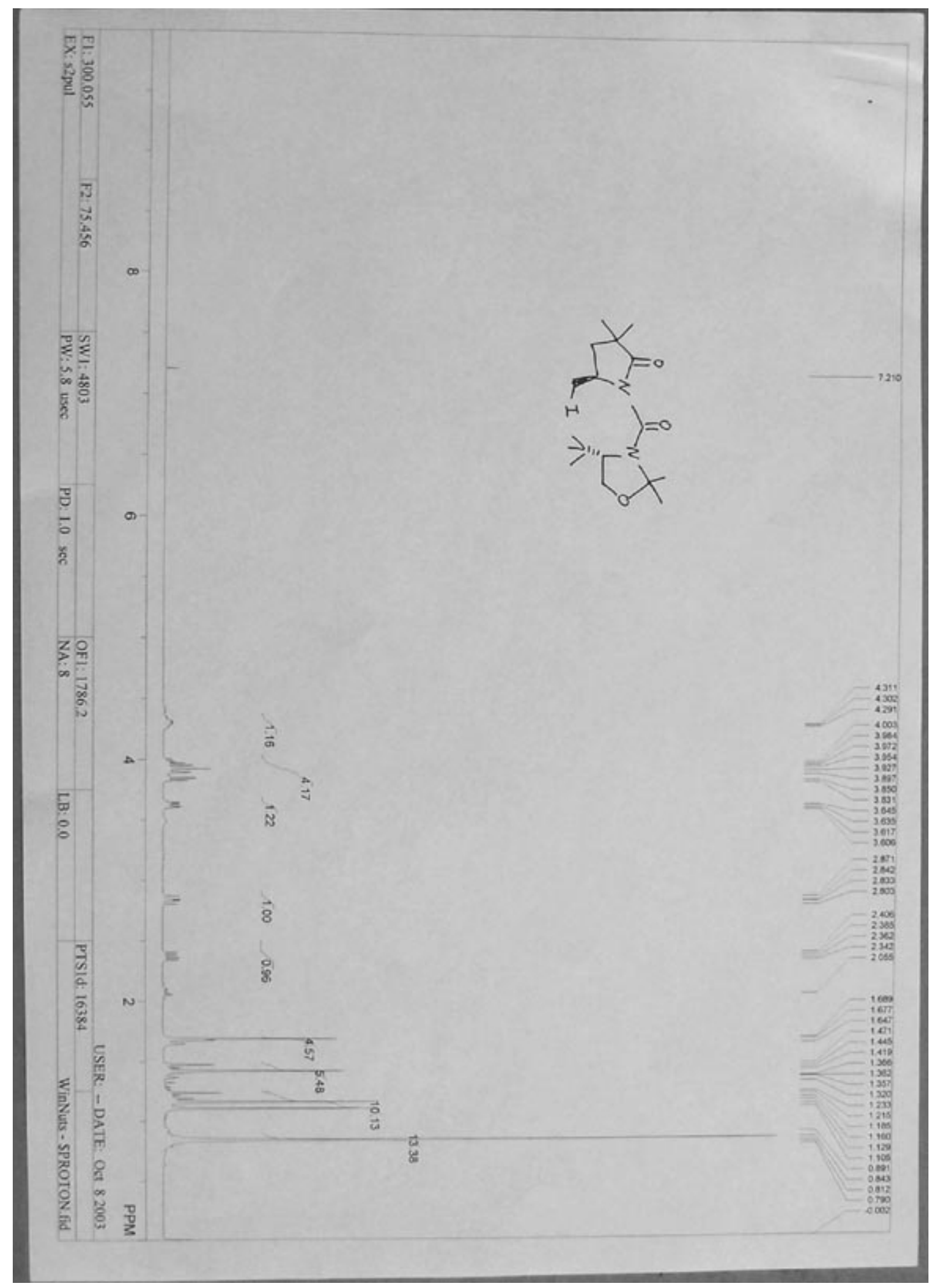




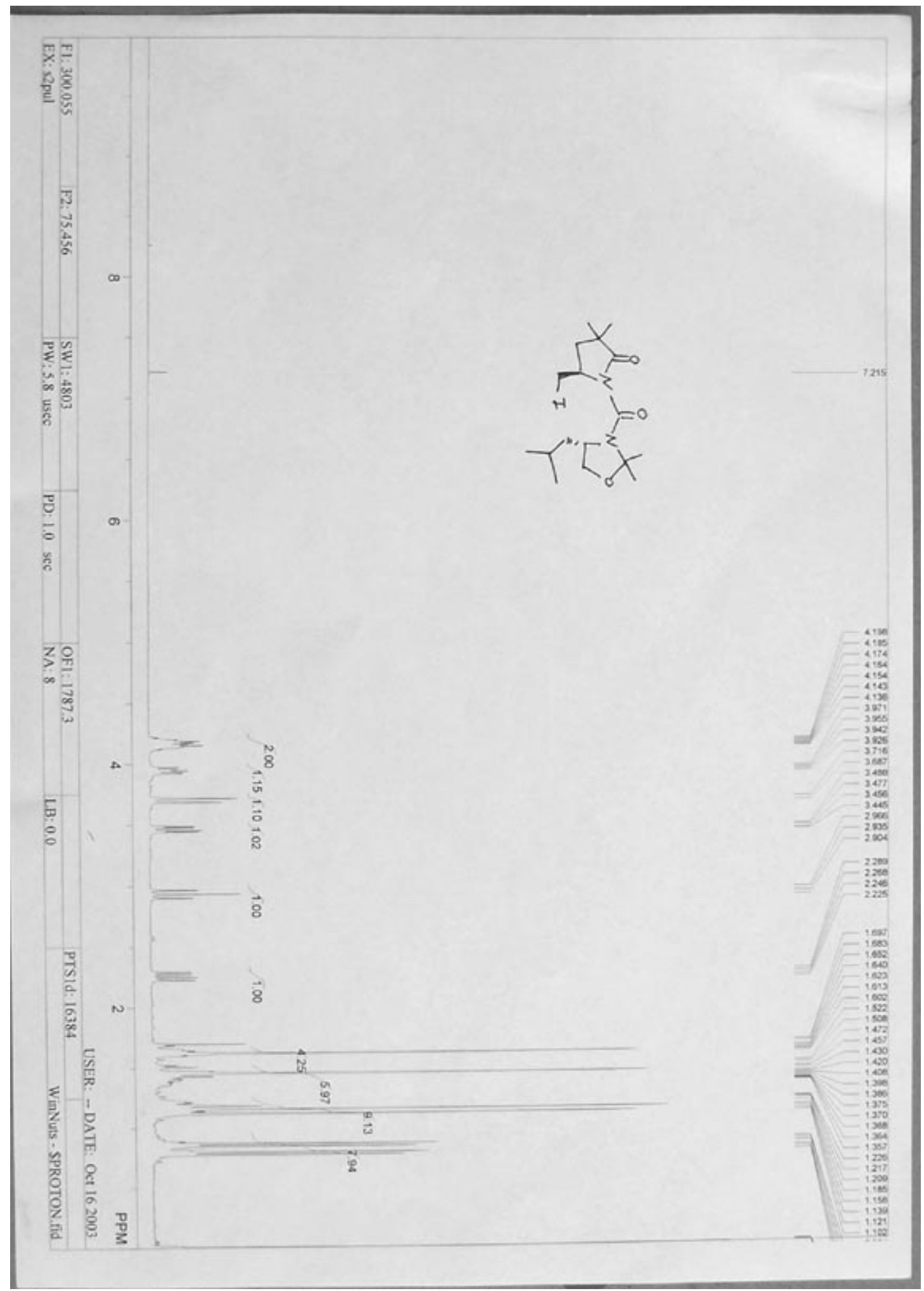




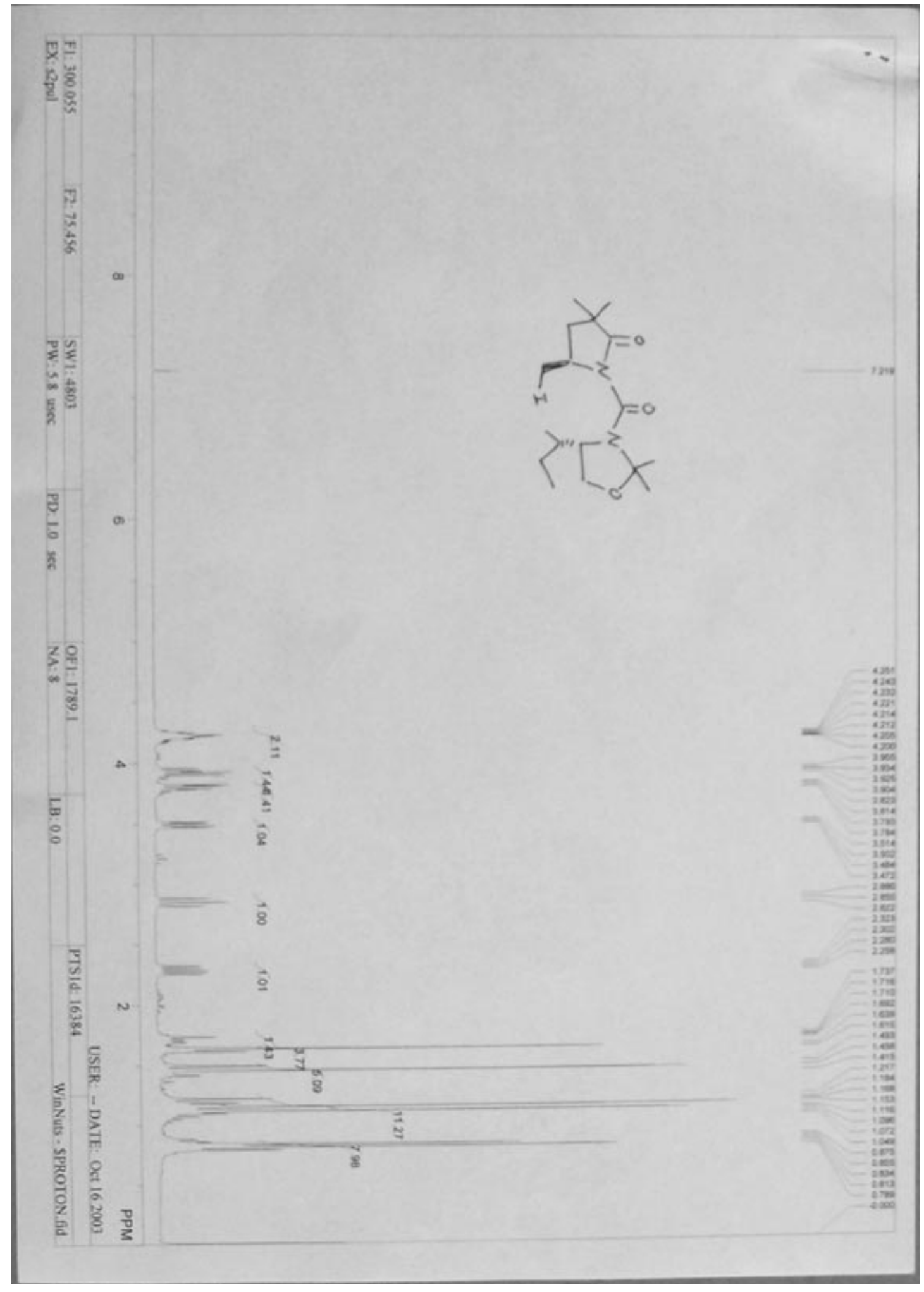




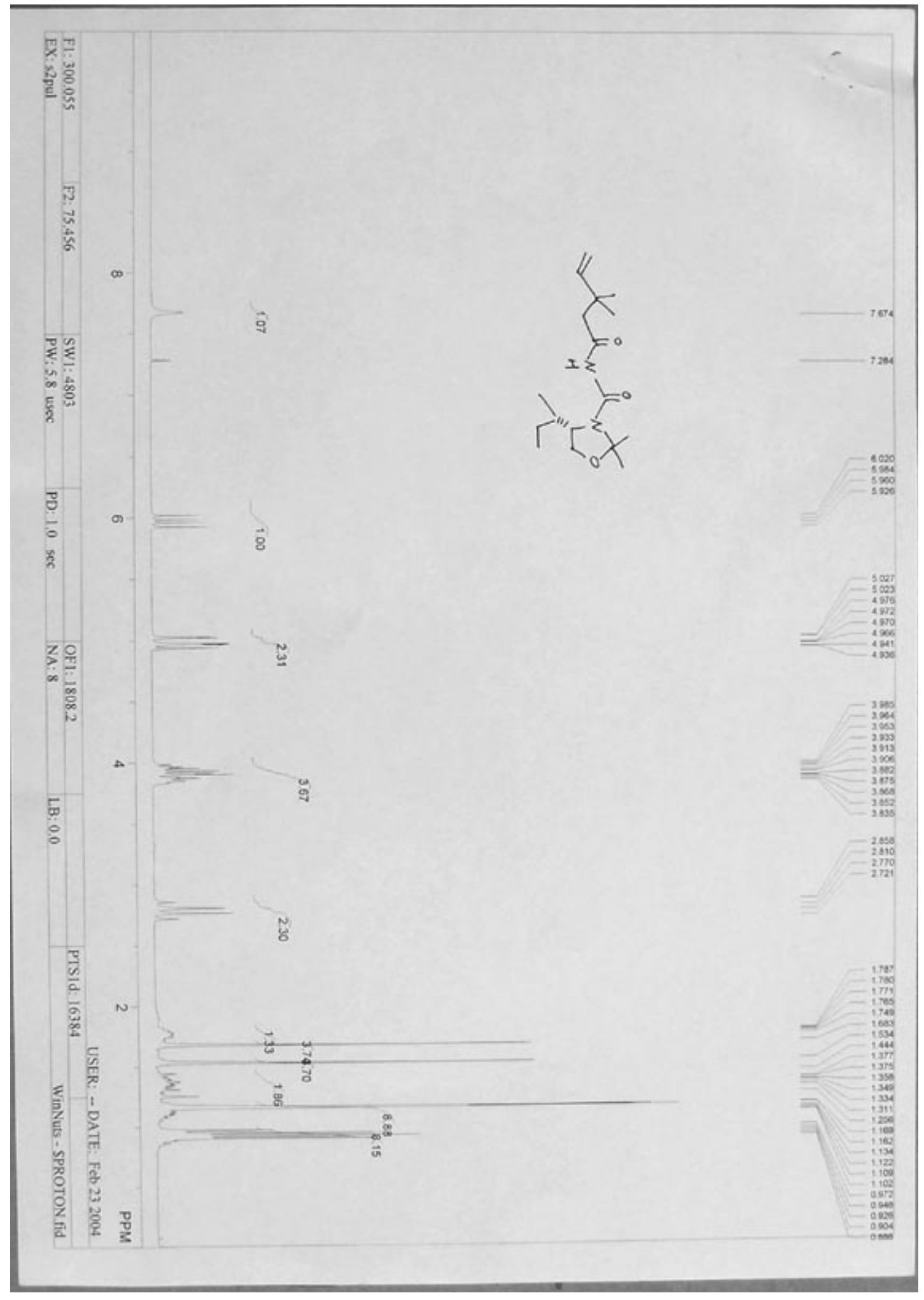




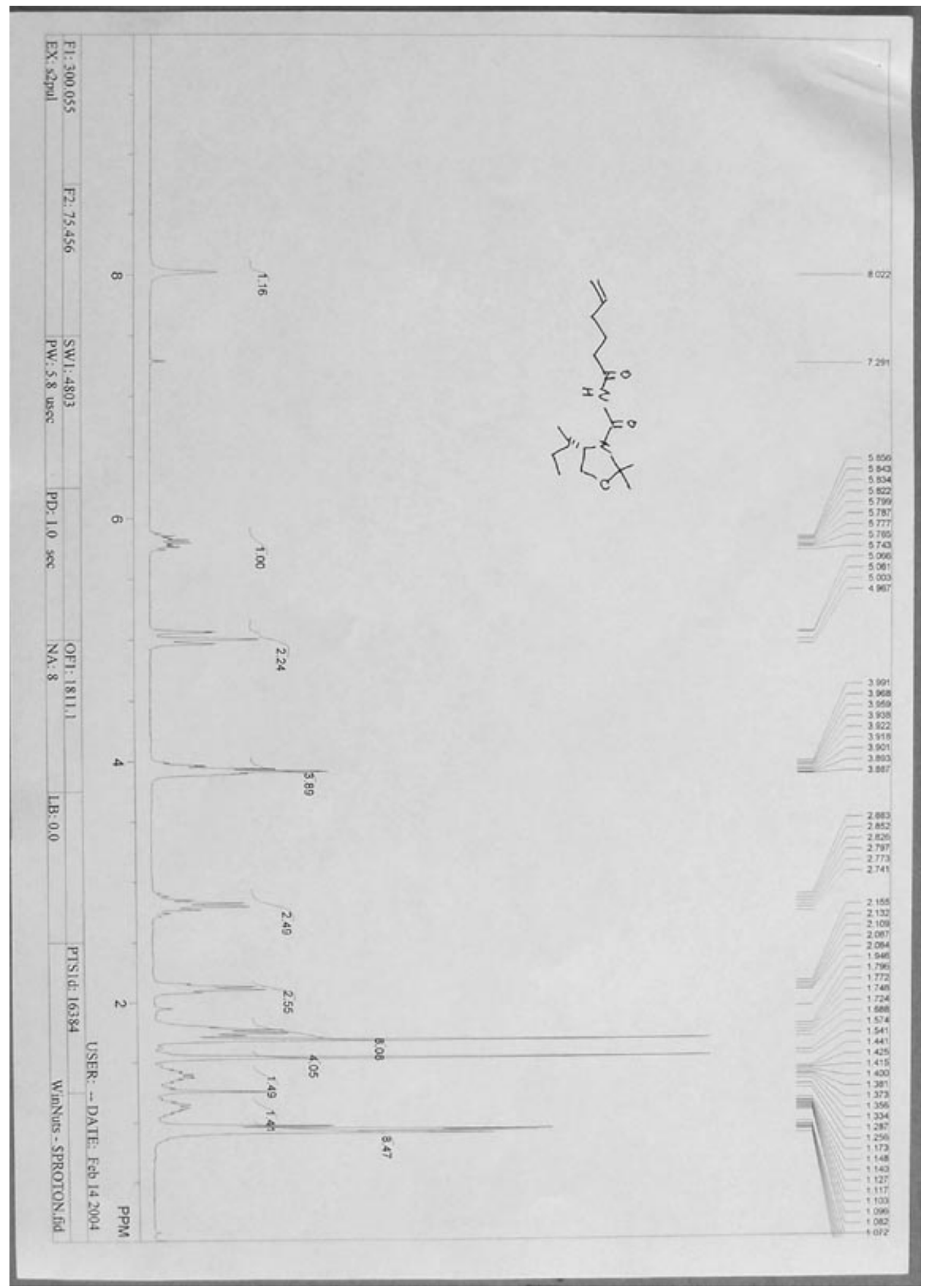




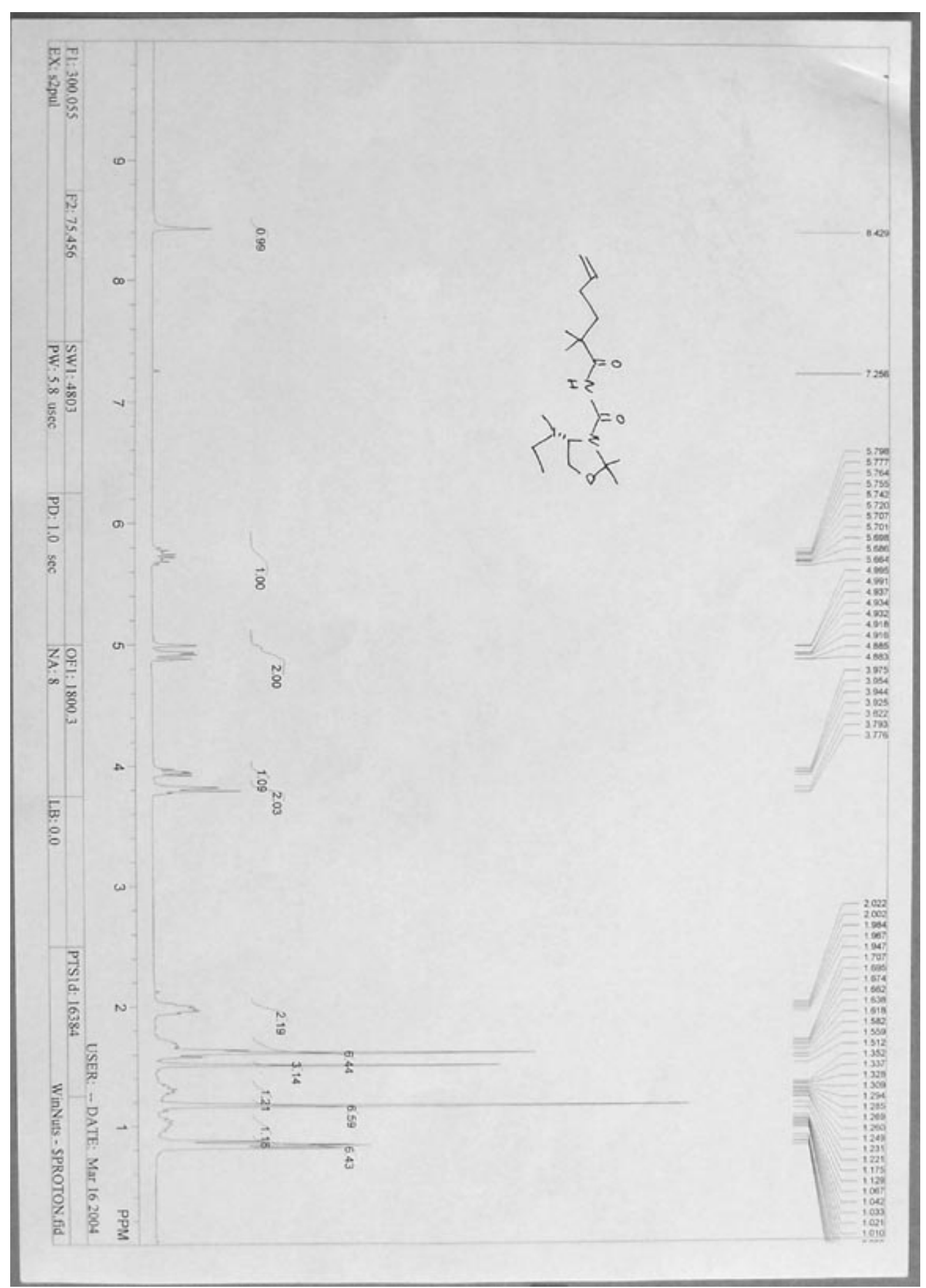




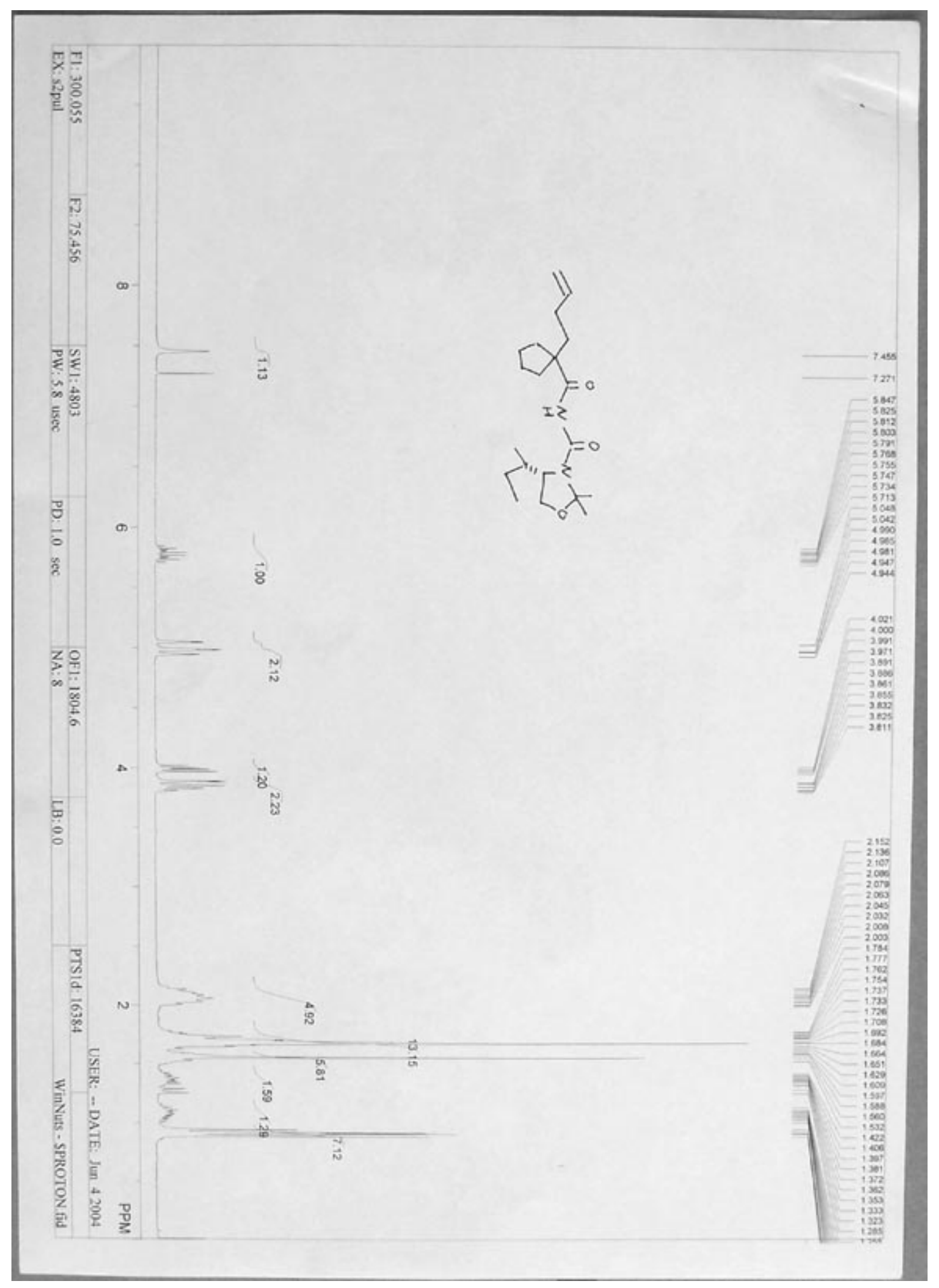




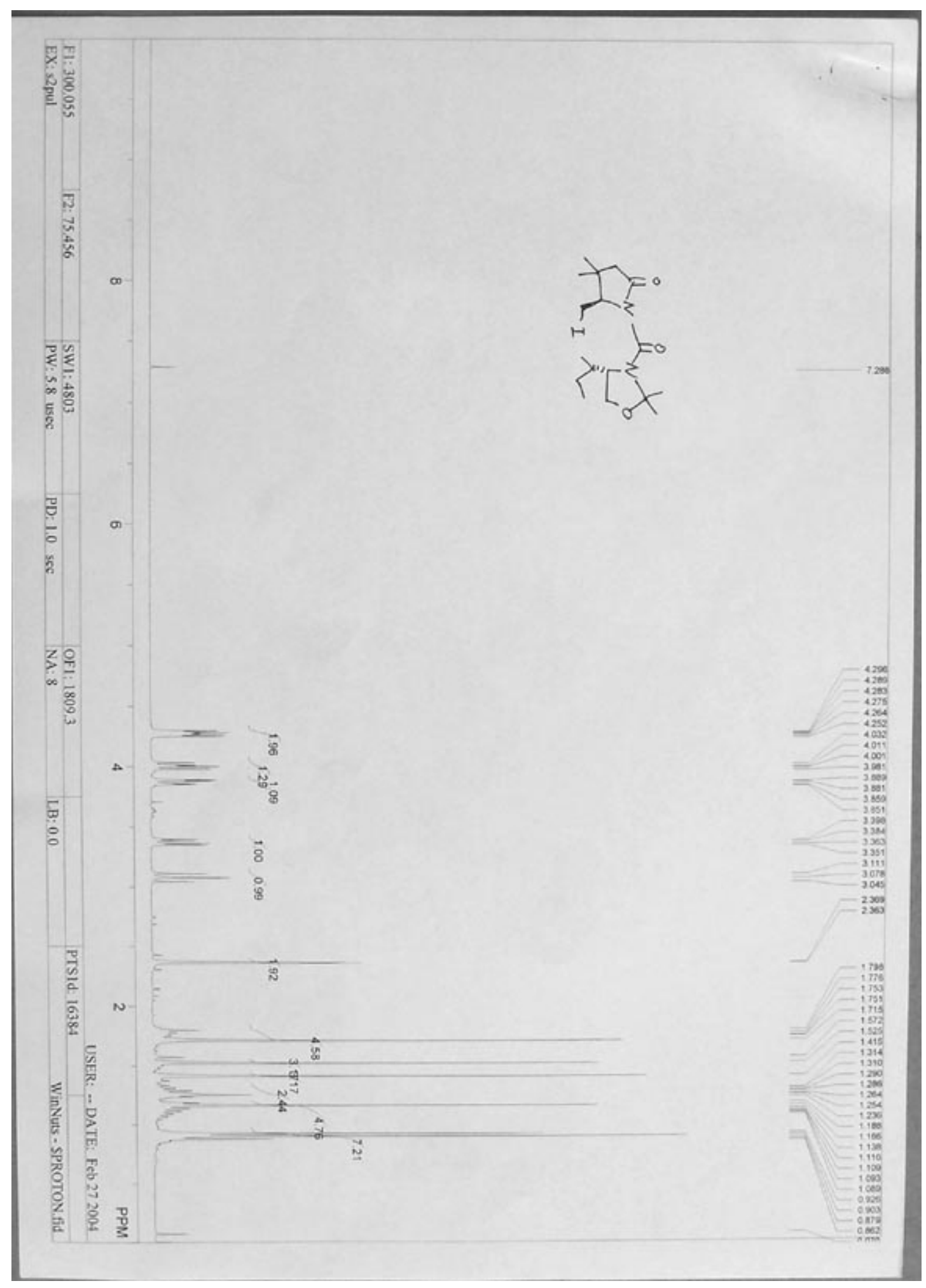




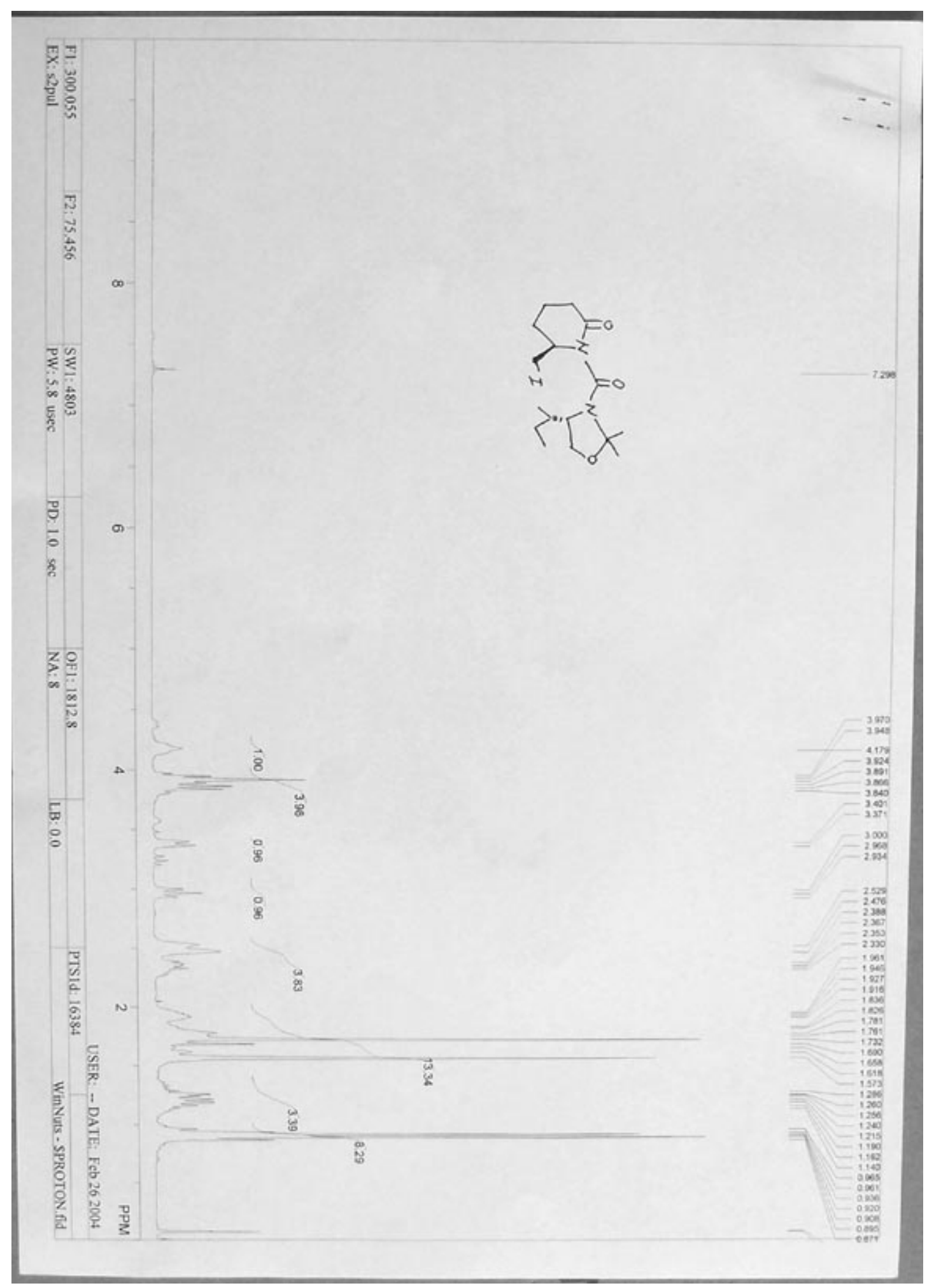




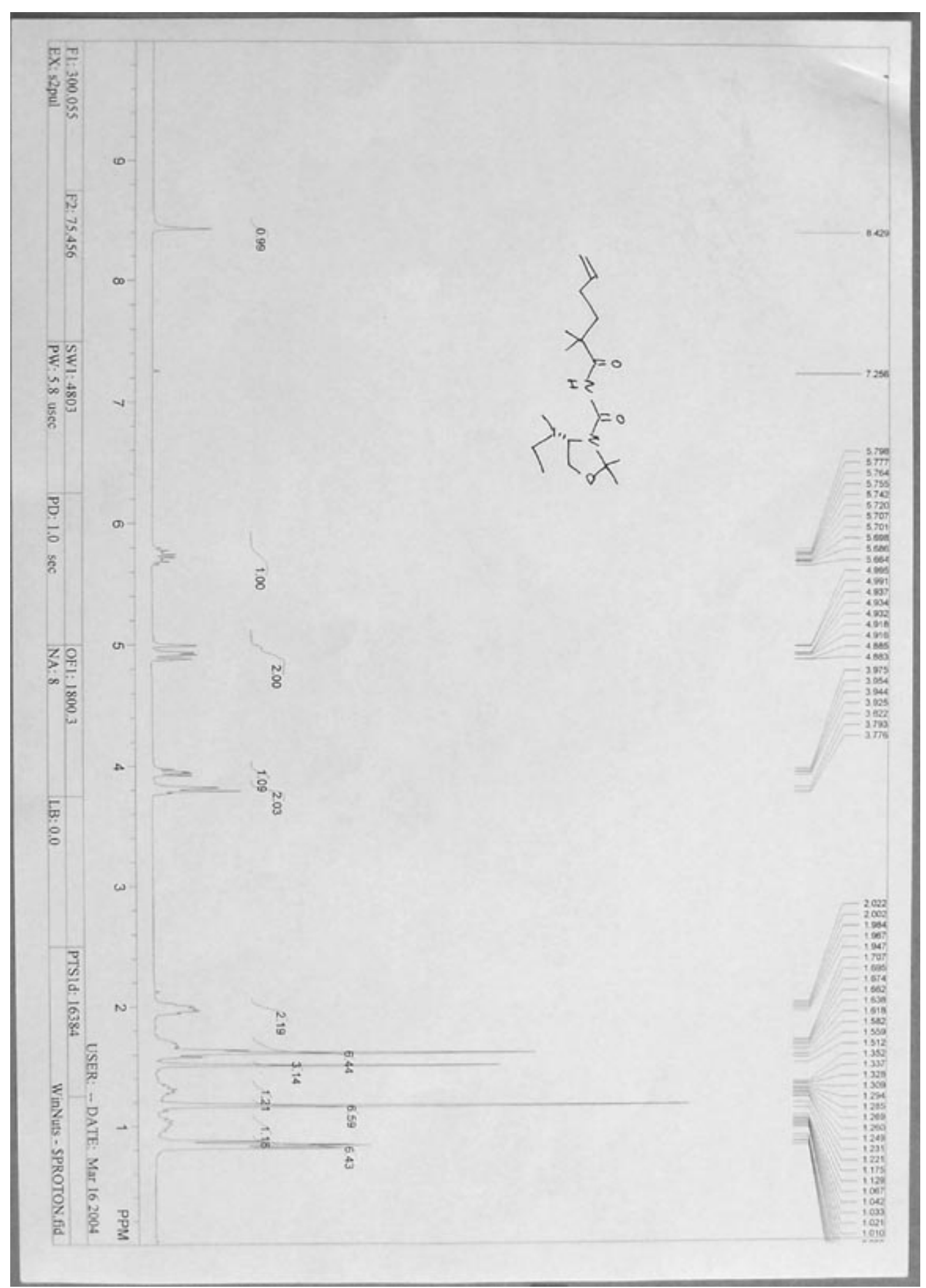


Figure 1. X-ray Crystal Structure of $6 \mathbf{c}$.

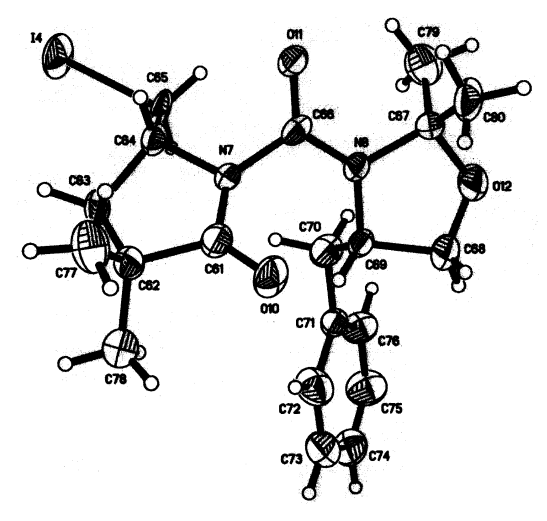

